\title{
A Phase II Study to Evaluate the Safety and Efficacy of Prasinezumab in Early Parkinson's Disease (PASADENA): Rationale, Design, and Baseline Data
}

\section{OPEN ACCESS}

Edited by:

Johannes Haybaeck,

Innsbruck Medical University, Austria

Reviewed by:

Santiago Perez-Lloret,

Consejo Nacional de Investigaciones

Cientificas y Técnicas

(CONICET), Argentina

Camila Aquino,

University of Calgary, Canada

*Correspondence:

Gennaro Pagano

gennaro.pagano@roche.com

Specialty section:

This article was submitted to

Movement Disorders,

a section of the journal

Frontiers in Neurology

Received: 05 May 2021

Accepted: 01 September 2021

Published: 01 October 2021

Citation:

Pagano G, Boess FG, Taylor KI, Ricci B, Mollenhauer B, Poewe W, Boulay A, Anzures-Cabrera J, Vogt A,

Marchesi M, Post A, Nikolcheva T,

Kinney GG, Zago WM, Ness DK,

Svoboda $H$, Britschgi $M$,

Ostrowitzki S, Simuni T, Marek K,

Koller M, Sevigny J, Doody R,

Fontoura $P$, Umbricht $D$, Bonni $A$ and

PASADENA Investigators and

Prasinezumab Study Group (2021) A

Phase II Study to Evaluate the Safety

and Efficacy of Prasinezumab in Early

Parkinson's Disease (PASADENA)

Rationale, Design, and Baseline Data.

Front. Neurol. 12:705407.

doi: 10.3389/fneur.2021.705407
Gennaro Pagano ${ }^{1 *}$, Frank G. Boess ${ }^{1}$, Kirsten I. Taylor ${ }^{1,2}$, Benedicte Ricci ${ }^{3}$, Brit Mollenhauer ${ }^{4,5}$, Werner Poewe ${ }^{6}$, Anne Boulay ${ }^{7}$, Judith Anzures-Cabrera ${ }^{8}$, Annamarie Vogt ${ }^{1}$, Maddalena Marchesi ${ }^{3}$, Anke Post ${ }^{9}$, Tania Nikolcheva ${ }^{10}$, Gene G. Kinney ${ }^{11}$, Wagner M. Zago ${ }^{11}$, Daniel K. Ness ${ }^{11}$, Hanno Svoboda ${ }^{1}$, Markus Britschgi ${ }^{1}$, Susanne Ostrowitzki ${ }^{10}$, Tanya Simuni ${ }^{12}$, Kenneth Marek ${ }^{13}$, Martin Koller ${ }^{11}$, Jeff Sevigny ${ }^{14}$, Rachelle Doody ${ }^{10}$, Paulo Fontoura ${ }^{10}$, Daniel Umbricht ${ }^{1}$, Azad Bonni ${ }^{1}$ and PASADENA Investigators and Prasinezumab Study Group

${ }^{1}$ Roche Pharma Research and Early Development (PRED), Neuroscience and Rare Diseases Discovery and Translational Area, Roche Innovation Center, F. Hoffmann-La Roche Ltd., Basel, Switzerland, ${ }^{2}$ Faculty of Psychology, University of Basel, Basel, Switzerland, ${ }^{3}$ Roche Pharma Research and Early Development (pRED), Pharmaceutical Sciences, Roche Innovation Center, F. Hoffmann-La Roche Ltd., Basel, Switzerland, ${ }^{4}$ Paracelsus-Elena-Klinik, Kassel, Germany, ${ }^{5}$ Department of Neurology, University Medical Center Göttingen, Göttingen, Germany, ${ }^{6}$ Department of Neurology, Innsbruck Medical University, Innsbruck, Austria, ${ }^{7}$ Idorisa Pharmaceuticals Ltd., Allschwil, Switzerland, ${ }^{8}$ Roche Products Ltd., Welwyn Garden City, United Kingdom, ${ }^{9}$ Feetme SAS, Paris, France, ${ }^{10} \mathrm{~F}$. Hoffmann-La Roche Ltd., Basel, Switzerland, ${ }^{11}$ Prothena Biosciences Inc., South San Francisco, CA, United States, ${ }^{12}$ Department of Neurology, Northwestern University Feinberg School of Medicine, Chicago, IL, United States, ${ }^{13}$ Institute for Neurodegenerative Disorders, New Haven, CT, United States,

${ }^{14}$ Prevail Therapeutics, New York, NY, United States

Background: Currently available treatments for Parkinson's disease (PD) do not slow clinical progression nor target alpha-synuclein, a key protein associated with the disease.

Objective: The study objective was to evaluate the efficacy and safety of prasinezumab, a humanized monoclonal antibody that binds aggregated alpha-synuclein, in individuals with early PD.

Methods: The PASADENA study is a multicenter, randomized, double-blind, placebocontrolled treatment study. Individuals with early PD, recruited across the US and Europe, received monthly intravenous doses of prasinezumab $(1,500$ or $4,500 \mathrm{mg})$ or placebo for a 52-week period (Part 1), followed by a 52-week extension (Part 2) in which all participants received active treatment. Key inclusion criteria were: aged 40-80 years; Hoehn \& Yahr (H\&Y) Stage I or II; time from diagnosis $\leq 2$ years; having bradykinesia plus one other cardinal sign of PD (e.g., resting tremor, rigidity); DAT-SPECT imaging consistent with PD; and either treatment naïve or on a stable monoamine oxidase $\mathrm{B}$ (MAO-B) inhibitor dose. Study design assumptions for sample size and study duration were built using a patient cohort from the Parkinson's Progression Marker Initiative (PPMI). In this report, baseline characteristics are compared between the treatmentnaive and MAO-B inhibitor-treated PASADENA cohorts and between the PASADENA and PPMI populations. 
Results: Of the 443 patients screened, 316 were enrolled into the PASADENA study between June 2017 and November 2018, with an average age of 59.9 years and 67.4\% being male. Mean time from diagnosis at baseline was 10.11 months, with $75.3 \%$ in H\&Y Stage II. Baseline motor and non-motor symptoms (assessed using Movement Disorder Society - Unified Parkinson's Disease Rating Scale [MDS-UPDRS]) were similar in severity between the MAO-B inhibitor-treated and treatment-naive PASADENA cohorts (MDS-UPDRS sum of Parts I + II + III [standard deviation (SD)]; 30.21 [11.96], 32.10 [13.20], respectively). The overall PASADENA population (63.6\% treatment naïve and $36.4 \%$ on MAO-B inhibitor) showed a similar severity in MDS-UPDRS scores (e.g., MDSUPDRS sum of Parts I + II + III [SD]; 31.41 [12.78], 32.63 [13.04], respectively) to the PPMI cohort (all treatment naïve).

Conclusions: The PASADENA study population is suitable to investigate the potential of prasinezumab to slow disease progression in individuals with early PD.

Trial Registration: NCT03100149.

Keywords: Parkinson's disease, alpha-synuclein ( $\alpha$-syn), prasinezumab, monoclonal antibodies, disease progression, MDS-UPDRS = Movement Disorder Society-Unified Parkinson's Disease Rating Scale, Phase II clinical trial, disease modification treatments

\section{INTRODUCTION}

There is a high medical need to develop long-lasting therapies that can affect the underlying cause of Parkinson's disease (PD) and, therefore, slow disease progression (1-3). Currently available treatments have powerful symptomatic effects, particularly on motor symptoms, but they do not address the pathological processes underlying the disease and do not prevent or slow clinical decline $(2,3)$. With the progressive loss of dopaminergic and non-dopaminergic neurons and synapses, available therapies gradually become less effective at controlling PD motor symptoms (4-6). Individuals with PD will invariably develop motor complications and lose their autonomy, adversely affecting their quality of life and placing a significant burden on caregivers, family members and healthcare systems (7-9). Despite the high prevalence and impact of non-motor symptoms on the quality of life of individuals with PD, treatment options for these symptoms are limited (10). A therapy that targets the underlying cause of the disease has the potential to slow motor progression as well as address non-motor symptoms $(11,12)$.

Postmortem findings suggest that the loss of dopaminergic neurons is accompanied spatially and temporally by the progressive development of intraneuronal Lewy pathology, which is a neuropathological hallmark of PD in distinct brain regions (13-17). Lewy pathology is abnormally enriched in alpha-synuclein, a protein with key functions in neurons $(17,18)$. Although the etiology of PD is yet to be elicited, the spatio-temporal association between Lewy pathology and neurodegeneration, together with evidence from in vitro and in vivo models, suggests that pathologically aggregated forms of alpha-synuclein may contribute to axonal and neuronal damage, formation of Lewy pathology and consequent neuronal loss and disease progression (18-22).
Preclinical findings in cellular and animal models also support the hypothesis that certain aggregated forms of alpha-synuclein may be taken up by neurons and may induce the formation of intracellular alpha-synuclein inclusions in PD (20, 23-25). The appearance of intraneuronal inclusions throughout the central and peripheral nervous systems may arise upon propagation of Lewy pathology from neuron to neuron in a concerted manner by extracellular transfer of aggregated alpha-synuclein $(21,26-28)$.

Clinical evidence also supports the hypothesis that alphasynuclein is a key driver in the etiology of PD. For instance, both missense mutations (29) and increased production of alphasynuclein due to duplication or triplication of the alpha-synuclein gene (SNCA) (30-32) cause early-onset autosomal dominant PD, with virtually $100 \%$ penetrance (33). Although the exact pathophysiological mechanism in these genetic causes remains unclear, aggregation of alpha-synuclein due to missense mutation or overexpression is supposed to drive disease onset and progression (34). Direct transfer of aggregated alpha-synuclein from neuron to neuron has not been directly observed in humans. However, embryonic dopaminergic neurons transplanted into the striatum of individuals with PD harbored inclusions reminiscent of Lewy pathology approximately a decade after initial grafting $(35,36)$ which, together with replicated observations in animal models $(23,24)$, suggests the possibility of intercellular propagation of Lewy pathology. In support of a caudo-rostral propagation of Lewy pathology, molecular imaging studies demonstrate damage or dysfunction of noradrenergic and serotonergic pathways prior to the dopaminergic pathways in prodromal idiopathic and SNCA genetic PD $(37,38)$.

The growing understanding of the role of alpha-synuclein in the development of Lewy pathology and the pathogenesis of PD support the rationale that targeting alpha-synuclein may have therapeutic potential (39). Preclinical in vivo models of 
alpha-synucleinopathy, such as transgenic mice overexpressing wild-type human alpha-synuclein or that develop pathology upon intracerebral injection of aggregated recombinant alphasynuclein, are valuable when studying drug mechanisms targeting alpha-synuclein. These models may help identify the downstream mode of action of therapeutic compounds. Indeed, neuropathological and behavioral deterioration in various mouse models of alpha-synuclein pathology was shown to be ameliorated by treatment with monoclonal antibodies binding to alpha-synuclein (12, 40-44).

Prasinezumab (previously known as RO7046015/PRX002) is an investigational, humanized monoclonal immunoglobulin G1 antibody directed against an epitope in the carboxyl terminus of human alpha-synuclein $(11,40,41,45)$. It binds to human aggregated alpha-synuclein with a high affinity and avidity $(11,40,41)$. Preclinical pharmacologic studies to evaluate efficacy and potency of the murine form of prasinezumab (9E4) were performed in two transgenic mouse lines featuring alpha-synuclein aggregation disorders: Line $\mathrm{D}$ and Line 61 mice. The mice were treated with weekly intraperitoneal administration of 9E4 or certain other alpha-synuclein antibodies over 5-6 months and showed reduced neuronal and synaptic loss and a reduction in intraneuronal build-up of alphasynuclein pathology (measured as alpha-synuclein inclusions in cortical and subcortical regions), reduction of gliosis, and an improvement in both cognitive and motor behaviors (12, 40-42).

Although blockade of cell-to-cell transmission of alphasynuclein by extracellular neutralization of pathogenic species has been proposed as the main mechanism of action of prasinezumab, preclinical experiments also suggest a potential engagement of the lysosomal pathway (12). Together, these preclinical data support the therapeutic potential of prasinezumab in slowing the progression of PD.

In a Phase I single-ascending-dose study in healthy volunteers and a Phase I multiple-ascending-dose study in individuals with $\mathrm{PD}$, prasinezumab was safe, able to penetrate the bloodbrain barrier (measured in the cerebrospinal fluid) and showed robust peripheral binding to alpha-synuclein $(11,45)$. Peripheral binding, measured as the lowering of circulating, free (unbound) serum alpha-synuclein, occurred within $1 \mathrm{~h}$ of administration of prasinezumab and was maintained for longer durations with higher doses of prasinezumab (11). Results also demonstrated a dose-dependent increase of prasinezumab in cerebrospinal fluid concentration, which was $\sim 0.3 \%$ relative to serum across all dose groups (11). Prasinezumab has a high apparent binding affinity to aggregated alpha-synuclein, which makes it bind more selectively to aggregated alpha-synuclein over monomeric alphasynuclein (11). Together with the observed cerebrospinal fluid concentrations achieved, it is predicted that $>90 \%$ of aggregated alpha-synuclein will be engaged by prasinezumab in the brain of individuals with PD at doses $\geq 1,500 \mathrm{mg}$ (11). The 1,500 and $4,500 \mathrm{mg}$ doses were selected as both were expected to saturate the target in a Phase II study in individuals with early PD.

Here we report the study rationale, design, and baseline patient characteristics of PASADENA, a Phase II clinical trial testing efficacy and safety of prasinezumab in individuals with early PD. The study design and assumptions for sample size and study duration were built, in part, using a patient cohort from the Parkinson's Progression Markers Initiative (PPMI) study group. The PPMI is a landmark, global, observational clinical study of individuals with PD designed to comprehensively evaluate cohorts of significant interest using advanced imaging, biological sampling and clinical and behavioral assessments to identify biomarkers of PD progression (46). We compared the baseline characteristics of the treatment-naive and the monoamine oxidase B (MAO-B) inhibitor-treated cohorts of the PASADENA population. We also compared the baseline characteristics of the total PASADENA population with the characteristics of a subset of individuals with early PD enrolled in the PPMI study, which was selected using similar inclusion criteria to the PASADENA study (47).

\section{METHODS \\ Study Design}

The Phase II study of Anti alpha-Synuclein AntiboDy in Early ParkiNson's diseAse (PASADENA) to evaluate the safety and efficacy of prasinezumab (NCT03100149) is a multicenter, randomized, double-blind, placebo-controlled study across $\sim 60$ sites in the United States, France, Austria, Germany, and Spain. The study was designed to evaluate the efficacy and safety of intravenous prasinezumab (received every 4 weeks) in participants with early-stage PD (Hoehn and Yahr [H\&Y] Stages I-II, time since diagnosis $\leq 2$ years).

The study consists of two parts: a 52-week, double-blind, placebo-controlled treatment period (Part 1), followed by a $52-$ week extension period during which all participants received active treatment but remained blinded to original dose allocation (Part 2) (Figure 1). A 12-week safety follow-up was mandatory for all participants, regardless of whether cessation of treatment occurred after Part 1 or Part 2.

In Part 1, participants were randomized with a 1:1:1 allocation ratio to either placebo, a high dose $(4,500 \mathrm{mg}$ for body weight $\geq 65 \mathrm{~kg} ; 3,500 \mathrm{mg}$ for body weight $<65 \mathrm{~kg}$ ) or a low dose (1,500 mg for all body weights) of prasinezumab. Body weight influences clearance (and volume of distribution) such that exposure increases in patients with lower body weight. Therefore, the use of an $\sim 25 \%$ lower dose in participants with lower body weight $(<65 \mathrm{~kg})$ is implemented at high doses where there is an increased risk for infusion-related reactions. To reduce the risk of infusion-related reactions, participants in the highdose group received a 2,000 mg intravenous infusion on Day 1 followed by an up-titration to the full dose on Day 28 which they then received every 4 weeks. In addition, the first three study treatment infusions (irrespective of treatment allocation) were prolonged to $2 \mathrm{~h}$ and were preceded by pre-medication with nonsedating antihistamine and acetaminophen (11). Randomization was stratified by sex, age group ( $<60$ vs. $\geq 60$ years) and use of MAO-B inhibitor at baseline (yes vs. no).

Participants from Part 1 were eligible to continue to Part 2 provided dopamine transporter with single-photon emission computerized tomography (DaT-SPECT) and magnetic resonance imaging (MRI) scans had been completed at screening 


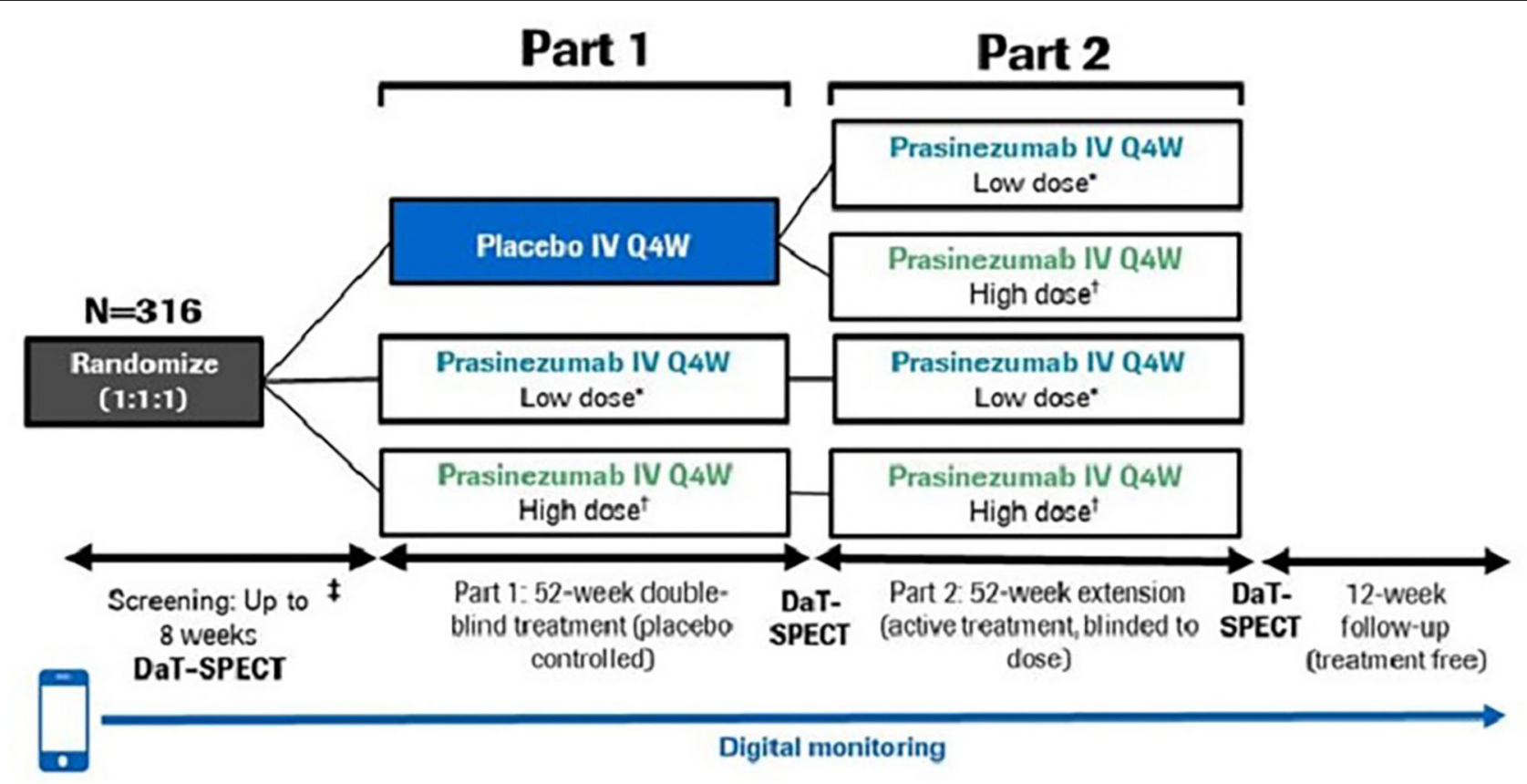

FIGURE 1 | PASADENA study design schematic. *Low dose =1,500 mg, ${ }^{\dagger}$ High dose = 4,500 mg for $\geq 65 \mathrm{~kg}$; 3,500 mg for <65 kg. DaT-SPECT, dopamine transporter imaging with single-photon emission computerized tomography; IV, intravenous; Q4W, every month.

and Week 52, and participants had received at least 10 doses of study treatment (placebo or prasinezumab) during Part 1.

In Part 2, participants randomized to treatment with prasinezumab in Part 1 remained on the same dose for the duration of Part 2. Those participants initially randomized to placebo were re-randomized to either 1,500 or $4,500 \mathrm{mg}$ prasinezumab using a 1:1 allocation ratio. Randomization was stratified by dopaminergic therapy since start of study (yes vs. no), sex, age group at start of study ( $<60$ vs. $\geq 60$ years) and use of MAO-B inhibitor at baseline (yes vs. no).

If symptomatic PD treatment was initiated during Part 1, investigators were required to record the reason(s) and the type and dose of symptomatic PD treatment prescribed. Participants who initiated symptomatic PD treatment could then continue in the study, as per their regular scheduled study visits. For participants who started dopaminergic treatment (levodopa or dopamine agonist), the Movement Disorder Society-Unified Parkinson's Disease Rating Scale (MDS-UPDRS) including Part IV (motor assessment while on dopaminergic treatment) and digital biomarker in-clinic assessments at subsequent visits were performed in an "Off" state, i.e., patients had not received levodopa since the previous evening ( $>8 \mathrm{~h}$ prior). The MDSUPDRS Part III (motor assessment) was repeated at least $1 \mathrm{~h}$ after receiving levodopa in the clinic (while patients are in an "On" state), along with digital biomarker in-clinic assessments.

\section{Study Population}

The inclusion and exclusion criteria were developed to select an early PD population with a measurable and predictable rate of progression over a 1-year period.
Key inclusion criteria included: idiopathic PD with bradykinesia and one of the other cardinal signs of PD (resting tremor, rigidity) and no other known or suspected cause of PD; aged 40-80 years; a DaT-SPECT consistent with PD; body weight range of $\geq 45$ to $\leq 110 \mathrm{~kg}$ and a body mass index of $18-34 \mathrm{~kg} / \mathrm{m}^{2}$; and either treatment naïve or on a stable dose of a MAO-B inhibitor for at least 90 days.

Key exclusion criteria included: medical history indicating a Parkinson syndrome other than idiopathic PD; known carriers of certain familial PD genes (Parkin, PINK1, DJ1); Mini Mental State Examination (MMSE) $\leq 25$; use of any of the following: catechol-O-methyl transferase inhibitors (entacapone, tolcapone), amantadine or anticholinergics, or dopaminergic medication (levodopa and both ergot and nonergot [pramipexole, ropinirole, rotigotine] dopamine agonists) for more than a total of 60 days or within 60 days of baseline and prior participation in any prasinezumab study.

A full list of inclusion and exclusion criteria can be found in Supplementary Tables 1, 2 .

\section{Objectives and Endpoints}

Clinical assessments performed at baseline and at different study visits are summarized in Figures 2, 3. A full list of endpoints is available online in the PASADENA study protocol (NCT03100149) (48).

\section{Primary Endpoint}

The primary objective of the study was to assess the efficacy of prasinezumab 1,500 and 4,500 mg vs. placebo at Week 52 in enrolled participants. The primary endpoint was the change from 


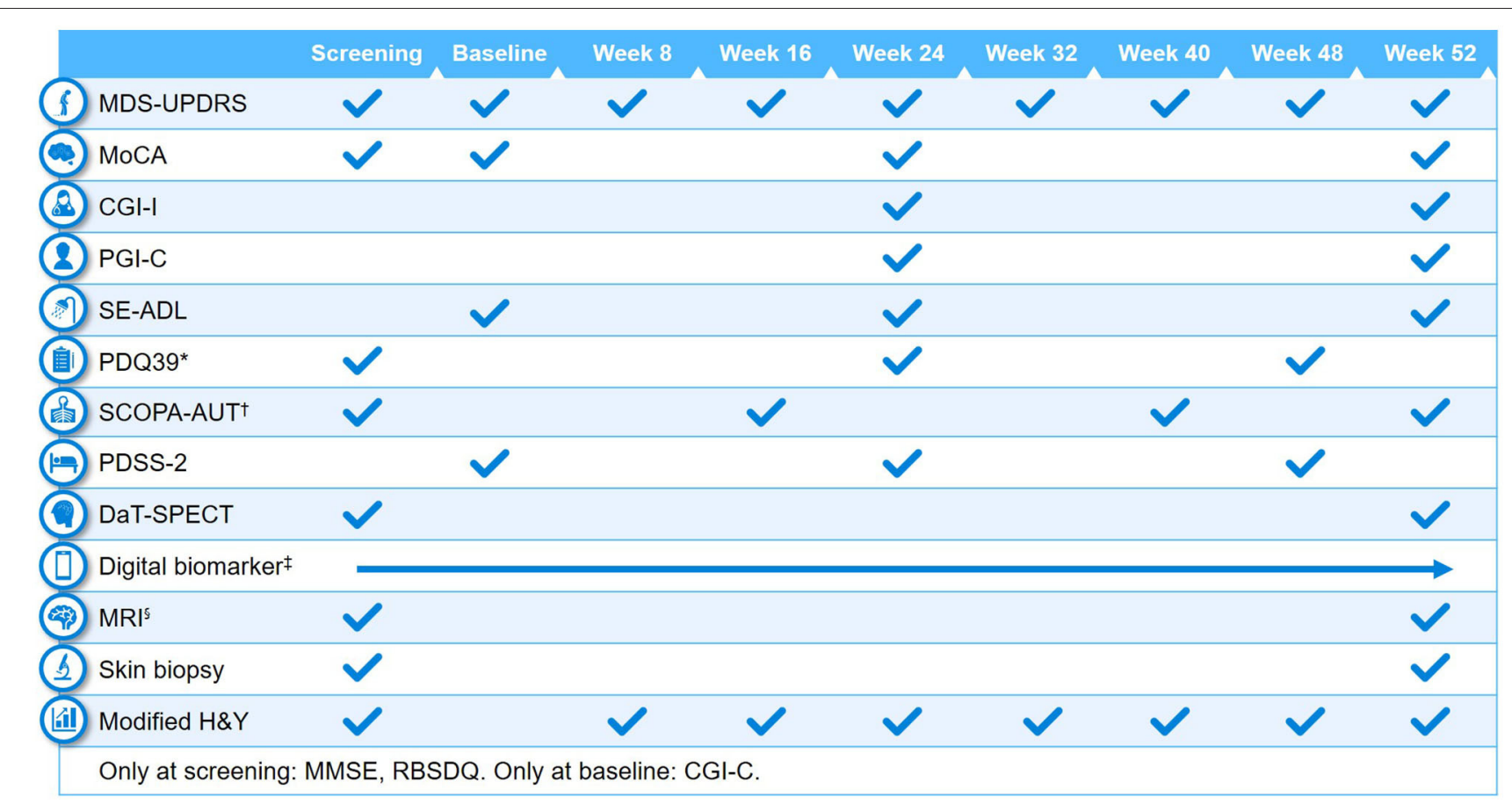

FIGURE 2 | Schedule of activities in PASADENA Part 1. *Parkinson's Disease Questionnaire-39 (PDQ39) is at baseline, Week 20, and Week 48; †SCOPA-AUT (Scales for Outcomes in Parkinson's Disease-Autonomic Dysfunction) is at baseline, Weeks 16, 28, 40 and 52. ${ }^{*}$ Digital includes PASADENA Digital Motor Score, Patient Global Impression of Severity, Daily diary, Patient Assessment of Constipation Symptoms (PAC-SYM), EuroQol-5D (EQ-5D) and Hospital Anxiety and Depression Scale (HADS). ${ }^{\circledR}$ Magnetic resonance imaging (MRI) includes safety, diffusion tensor imaging (DTI), resting state and arterial spin labeling (ASL). CGI-C, Clinical Global Impression of Change; CGI-I, Clinical Global Impression of Improvement; DaT-SPECT, dopamine transporter imaging with single-photon emission computerized tomography; H\&Y, Hoehn \& Yahr; MDS-UPDRS, Movement Disorder Society -Unified Parkinson's Disease Rating Scale; MMSE, Mini Mental State Examination; MoCA, Montreal Cognitive Assessment; PDSS-2, Parkinson's Disease Sleep Scale Revised Version 2; PGI-C, Patient Global Impression of Change; RBDSQ, Rapid Eye Movement Sleep Behavior Disorder Screening Questionnaire; SE-ADL, Schwab and England Activities of Daily Living.

baseline at Week 52 in MDS-UPDRS sum of Parts I + II + III vs. placebo.

\section{Secondary Endpoints}

The effects of prasinezumab 1,500 and $4,500 \mathrm{mg}$ vs. placebo at Week 52 on MDS-UPDRS Part IA, Part IB, Part I total, Part II total, Part III total, and Part III subscores (bradykinesia, rigidity, resting tremor, and axial symptoms) were included as secondary endpoints. Part I assessed non-motor experiences of daily living, with Part IA focused on complex behaviors (cognitive impairment, hallucinations, and psychosis, etc.) and Part IB focused on non-motor experiences (sleep and urinary problems, constipation, pain, etc.). Part II assessed motor experiences of daily living (eating, dressing, handwriting, getting out of bed, etc.) and Part III assessed motor signs of PD (speech, finger tapping, bradykinesia, gait, and freezing, etc.) (49). Part IA and Part III were administered by the study investigator, and Part IB and Part II were completed by the participant.

Other secondary endpoints included; Montreal Cognitive Assessment (MoCA) Total score; Clinical Global Impression of Improvement (CGI-I); Patient Global Impression of Change (PGI-C); DaT-SPECT in the ipsilateral (to the clinically dominant side) putamen; Schwab and England Activities of Daily Living
(SE-ADL) score; time to worsening in motor or non-motor symptoms (increase of $\geq 3$ points in MDS-UPDRS Part I or MDS-UPDRS Part II); time to start of dopaminergic PD treatment (levodopa or dopamine agonists); and safety (Supplementary Table 3), tolerability, immunogenicity, and pharmacokinetics (PK) of prasinezumab. Safety and tolerability were assessed for up to 104 weeks, with or without dopaminergic treatment. PK of prasinezumab was assessed using population PK modeling.

\section{Exploratory Endpoints}

Exploratory endpoints of this study included: MDS-UPDRS Part III subscores determined by independent central raters (using video recordings to address consistency and accuracy in the trained site raters), imaging analysis of striatum, caudate, and putamen (average, ipsilateral, and contralateral) for DaTSPECT binding ratio values, and the change from baseline on a sensor-based measure derived from Roche PD Mobile Application v2 digital biomarkers (50) (smartphone and wristworn wearable) assessments (see Figure 4 for an overview of the remote monitoring tests). The analyses of primary and secondary endpoints were also repeated, with the results for the two prasinezumab 1,500 and 4,500 $\mathrm{mg}$ pooled doses vs. placebo, as a pre-specified exploratory analysis. 


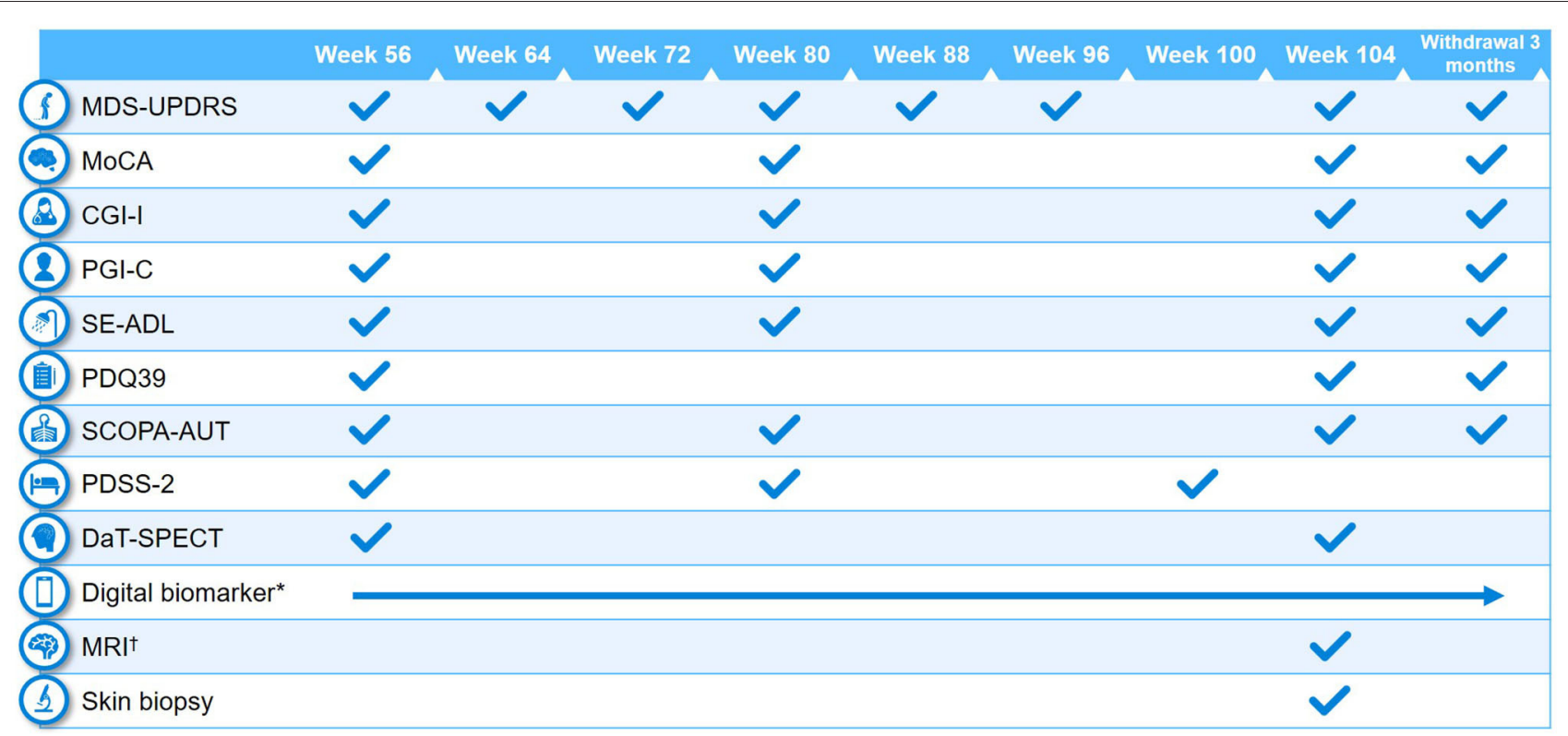

FIGURE 3 | Schedule of activities in PASADENA Part 2. *Digital includes PASADENA Digital Motor Score, Patient Global Impression of Severity, Daily diary, Patient Assessment of Constipation Symptoms (PAC-SYM), EuroQol-5D (EQ-5D), and Hospital Anxiety and Depression Scale (HADS). ${ }^{\dagger}$ Magnetic resonance imaging (MRI) includes safety, diffusion tensor imaging (DTI), resting state and arterial spin labeling (ASL). CGI-I, Clinical Global Impression of Improvement; DaT-SPECT, dopamine transporter imaging with single-photon emission computerized tomography; MDS-UPDRS, Movement Disorder Society-Unified Parkinson's Disease Rating Scale; MoCA, Montreal Cognitive Assessment; PDQ39, Parkinson's Disease Questionnaire-39; PDSS-2, Parkinson's Disease Sleep Scale Revised Version 2; PGI-C, Patient Global Impression of Change; SCOPA-AUT, Scales for Outcomes in Parkinson's Disease-Autonomic Dysfunction; SE-ADL, Schwab and England Activities of Daily Living.

\begin{tabular}{|c|c|c|c|c|c|c|c|c|c|c|c|c|c|c|}
\hline \multicolumn{10}{|c|}{ ACTIVE TESTS } & \multicolumn{3}{|c|}{ PASSIVEMONITORING } & \multicolumn{2}{|c|}{ IN-CLINICTESTS } \\
\hline \multicolumn{3}{|c|}{ Brodykinesio } & \multirow{2}{*}{$\begin{array}{c}\text { Tremor/ } \\
\text { Brodykinesia } \\
\text { Speech }\end{array}$} & \multicolumn{3}{|c|}{ Tremor } & \multicolumn{2}{|c|}{$\begin{array}{c}\text { Rigidity/ } \\
\text { Postural Instobility }\end{array}$} & \multirow{2}{*}{$\begin{array}{l}\text { cognition } \\
\text { Cognitive } \\
\text { Test } \\
\text { (SOMT) }\end{array}$} & \multicolumn{3}{|c|}{$\begin{array}{c}\text { Bradykinesia and Activities of Daily } \\
\text { Living }\end{array}$} & \multicolumn{2}{|c|}{ Balance } \\
\hline $\begin{array}{l}\text { Draw } \\
\text { A Shape }\end{array}$ & Dexterity & $\begin{array}{l}\text { Hand } \\
\text { Turning }\end{array}$ & & Phonation & $\begin{array}{l}\text { Postural } \\
\text { Tremor }\end{array}$ & Rest Tremor & Balance & U.Turn & & Gait & $\begin{array}{l}\text { Arm Swing } \\
\text { \& Tremor }\end{array}$ & $\begin{array}{l}\text { Mobility \& } \\
\text { Sociability }\end{array}$ & $\begin{array}{c}\text { Timed Up } \\
\text { \& Go }\end{array}$ & $\begin{array}{c}\text { Berg } \\
\text { Balance } \\
\text { Scale }\end{array}$ \\
\hline \multicolumn{3}{|c|}{$\begin{array}{l}\text { Bradykinesia Days } \\
\text { (Every } 2^{\text {st }} \text { Oay) }\end{array}$} & Altern & lating & \multicolumn{4}{|c|}{$\begin{array}{l}\text { Tremor and Stability Days } \\
\text { (Every } 2^{\text {ns }} \text { Day) }\end{array}$} & Fortnightly & Daily & Daily & Daily & $\begin{array}{c}\mathrm{At} \\
\text { selected } \\
\text { visits }\end{array}$ & $\begin{array}{l}\text { At } \\
\text { selected } \\
\text { visits }\end{array}$ \\
\hline
\end{tabular}

FIGURE 4 | Table of digital measures included in the Roche Parkinson's Disease Mobile Application v2. SDMT, Symbol Digit Modalities Test.

A full list of exploratory endpoints is included in Supplementary Table 4.

\section{PASADENA Digital Motor Score}

Summary scores of sensor data from digital health technology tools should be developed independent of existing clinical data to ensure that the digital score does not inherit the shortcomings of the clinical measure (e.g., restriction of range, reduced resolution of scale). However, this requires independent longitudinal sensor datasets to build and validate such a digital score (50). Since such data are not yet available, a provisional single summary sensorbased measure, the "PASADENA Digital Motor Score", reflecting global motor function was developed. Data from a PASADENAlike PPMI cohort were used to inform sensor feature selection for the PASADENA Digital Motor Score, as follows: MDS-UPDRS Parts II and III item-level scores which significantly declined in Year 1 of PPMI were identified [ $n=21$; whereby one item (posture) was not tested with the digital biomarker; final set of MDS-UPDRS items $=20$ ]. A blinded subset of PASADENA data $(n=157)$ was used to map sensor feature data (aggregated over 2-week periods) onto each MDS-UPDRS item with the blinded PASADENA population. The set of sensor features was used to predict the sum of the 20 identified MDS-UPDRS items. PASADENA Digital Motor Scores were generated for every 2 weeks of the study and submitted to linear random coefficient 
(i.e., slope) models testing for differences in slopes between censored patients in each treatment group over the 262 -week periods of the PASADENA study.

A second set of exploratory digital biomarker analyses comprised 17 individual pre-specified sensor features, which were selected based on a previous communication that reported cross-sectional correlations between sensor features and MDSUPDRS scores (51) and available literature. These individual sensor features were also analyzed with linear random coefficient models testing for differences in slopes across treatment groups if the model's residuals were normally distributed; if the residuals were not normally distributed, mixed-effect models for repeated measures (MMRM) were applied.

\section{Standard Protocol Approvals, Registrations, and Patient Consents}

This study was conducted in full conformance with the International Conference on Harmonization E6 guideline for Good Clinical Practice and the principles of the Declaration of Helsinki, or the laws and regulations of the country in which the research was conducted, whichever afforded the greater protection to the individual. The study protocol, Informed Consent Forms, and any information given to the participant, were approved by the Institutional Review Board or Ethics Committee (NCT03100149).

\section{Sample Size Justification}

A sample size of $\sim 100$ randomized participants per group (300 participants in total for the three groups) was estimated, which allowed for a power of $\sim 80 \%$ at two-sided $\alpha$-level of $20 \%$ to detect a three-point difference in MDS-UPDRS sum of Parts I + II + III between groups from baseline at Week 52. The 3-point difference was selected based on clinical judgement of expert consultants in movement disorders and modeling on PPMI data, which were used to model the disease progression of the placebo arm in the PASADENA study. The power calculation was based on simulations of the MMRM analysis planned for the primary efficacy variable. Assessments performed while on any dopaminergic PD treatment started after randomization were not included in the analysis of the primary endpoint.

The assumptions on progression, variability, dropout rate, and likelihood to start dopaminergic PD treatment within the first 52 weeks of treatment, with or without a MAO-B inhibitor as background therapy, were derived from analyses based on the PPMI database and various sources of information from the literature $(47,52)$. The percentage of patients defined as nonevaluable at Week 52 was predicted to be $25 \%$ in the placebo group and $20 \%$ in the treatment groups. It was expected that patients treated with prasinezumab would be less likely to start dopaminergic PD treatment during the study period, compared with those treated with placebo. This estimate included nonevaluable data from patients who prematurely dropped out and/or started dopaminergic PD treatment after randomization.

The sample size of 100 patients per arm also provided $76 \%$ power ( $\alpha=20 \%$, two sided) to reject the null hypothesis, assuming a $37.5 \%$ reduction for the key secondary endpoint, the
DaT-SPECT signal loss at Week 52 and the pairwise comparison of each active dose arm with placebo $(47,52)$.

\section{Covariate Adjustment}

Analyses of efficacy endpoints (primary, secondary, and exploratory) included the following covariates in the model: background therapy at baseline (MAO-B inhibitor treatment [yes vs. no]), age group ( $<60$ vs. $\geq 60$ years), sex (male vs. female), DaT-SPECT binding ratio in the contralateral (to the clinically most affected side) putamen at baseline, and treatment group (4,500 mg prasinezumab, 1,500 $\mathrm{mg}$ prasinezumab or placebo). For each continuous endpoint the baseline of the endpoint variable was included in the model.

\section{Statistical Analyses of Primary Efficacy Endpoint}

Change in MDS-UPDRS sum of Parts I + II + III from baseline vs. placebo was analyzed using an MMRM, with covariates described in the Covariate adjustment section as fixed effects. The model also included baseline MDS-UPDRS sum of Parts I + II + III, week of treatment (as a categorical factor), a treatment-byweek interaction term and an interaction term between baseline MDS-UPDRS by week. An unstructured variance-covariance matrix was used to model the random error. The model tested the null hypothesis of no treatment difference at a two-sided $\alpha$-level of $20 \%$ for the following comparisons:

- 4,500 or 3,500 mg (high dose) prasinezumab vs. placebo.

- $1,500 \mathrm{mg}$ (low dose) prasinezumab vs. placebo.

All patients randomized in the study were included in the analyses. All the assessments flagged on and after the "first symptomatic PD treatment date" (either initiation of symptomatic PD treatment or a change to MAO-B inhibitor dose) were not included in the analysis per the hypothetical strategy described in the ICH E9(R1) Addendum on Estimands and Sensitivity Analyses in Clinical Trials by the European Medicines Agency. The MMRM model does not perform an imputation of missing data; however, high correlations between successive observations of a subject allow data from subjects who started dopaminergic PD treatment to contribute to the estimation at their final time-point. The primary endpoint of MDS-UPDRS sum of Parts I + II + III was re-analyzed with data from the two treatment arms pooled and compared with placebo as an exploratory endpoint.

\section{Statistical Analyses of Secondary and Exploratory Efficacy Endpoints}

For the endpoints utilizing MDS-UPDRS Part IA, Part IB, Part I total, Part II total, Part III total, Part III subscores, CGII, and PGI-C, the information collected after symptomatic PD treatment was handled as for the primary analysis. The analysis of all other endpoints included all the data available regardless of start of symptomatic PD treatment.

An analysis of covariance (ANCOVA) was used to analyze the change from baseline at Week 52 in MoCA Total score and SE-ADL score with covariates described in the Covariate adjustment section. The change (between baseline and Week 52) in DaT-SPECT striatal binding ratio in the 
ipsilateral putamen to the clinically most affected side, was analyzed using ANCOVA. Arterial spin labeling (ASL) MRI was performed at sites with the technical capability. Effects are tested for in pre-specified regions of interest; striatum, caudate, and putamen (ipsilateral, contralateral, and average were assessed for each region). Between-group changes in ASL MRI over 1 year in regions of interest were also tested using ANCOVAs.

The CGI-I was intended as a measure of change in health status from baseline CGI-Severity of illness (CGI-S). For the CGI-I, patients were divided into one of two groups:

- "Responders": Score of 1-4 (i.e., rated as "no change," "minimally improved," "much improved," or "very much improved").

- "Progressors": Score of 5-7 (i.e., rated as "minimally worse," "much worse," or "very much worse").

The proportion of patients rated by CGI-I grouping at Week 24 and Week 52 was analyzed using a logistic regression model. The estimated odds ratio for "responders" and "progressors" at Week 24 and Week 52 for treated patients compared with placebo were calculated with $80 \%$ confidence interval. Analysis of the PGI-C followed the same methodology outlined for the CGI-I above.

Time to worsening of motor or non-motor symptoms (of $\geq 3$-point change from baseline in MDS-UPDRS Part I or Part II) and time to start of dopaminergic (levodopa or dopamine agonist) treatment were plotted using a Kaplan-Meier survival plot and analyzed using a Cox proportional hazards model to obtain a treatment difference between each of the prasinezumab dose levels against placebo. A 3-point minimum change in MDSUPDRS Part II was chosen as this has been previously identified as the smallest change of score that is clinically meaningful to patients (53).

\section{Subgroup Analyses}

Subgroups with $\geq 20 \%$ of patients from the modified intent-totreat population at baseline were analyzed. The model used for the primary endpoint was run in each subgroup, excluding the subgroup being analyzed if that was a covariate (e.g., MAO$B$ inhibitors at baseline [yes vs. no]). The subgroup analyses were performed in the primary endpoint and the following secondary/exploratory endpoints:

- MDS-UPDRS Part I

- MDS-UPDRS Part II

- MDS-UPDRS Part III Total score and subscores

- MDS-UPDRS sums of Part II and III

- DaT-SPECT striatal binding ratio in the ipsilateral putamen to the clinically most affected side

- PASADENA Digital Motor Score

- MoCA score

- Time to worsening in motor signs (increase of $\geq 3$ points in MDS-UPDRS Part III)

- Time to worsening in motor or non-motor symptoms (increase of $\geq 3$ points in MDS-UPDRS Part I or MDS-UPDRS Part II)
Subgroups included in analyses were:

- MAO-B inhibitors at baseline (yes vs. no)

- H\&Y Stage at baseline (I vs. II)

- Rapid Eye Movement Sleep Behavior Disorder Screening Questionnaire (RBDSQ) at baseline (RBDSQ $\geq 5$ vs. $<5$ )

- Data-driven subphenotypes (Diffuse malignant vs. mild motor predominant vs. intermediate) at baseline

- Alpha-synuclein skin (positive vs. negative) (staining by immunohistochemistry on skin biopsy sections at baseline)

- DaT-SPECT striatal binding ratio in the ipsilateral putamen to the clinically most affected side (very abnormal vs. abnormal).

For the derivations of the data-driven subphenotypes, scales were classified into: motor scales (MDS-UPDRS Part II and MDSUPDRS Part III) and non-motor scales (Scales for Outcomes in Parkinson's Disease-Autonomic Dysfunction [SCOPA-AUT], RBDSQ, and MoCA). After each one of the scales had been divided into percentiles, the data-driven subphenotypes were defined as follows:

- Diffuse malignant: Score on motor scales being greater than the $75^{\text {th }}$ percentile; and at least one score on a non-motor scale greater than the $75^{\text {th }}$ percentile; or all three non-motor scores greater than the $75^{\text {th }}$ percentile

- Mild motor predominant: Motor and all non-motor scores less than the $75^{\text {th }}$ percentile

- Intermediate: All those individuals not meeting criteria for other subtypes.

\section{Inclusion Criteria for the PPMI Cohort}

The design of this study and the assumptions for progression, variability, dropout rate, and likelihood to start symptomatic treatment within the first 52 weeks of treatment, with or without a MAO-B inhibitor, were derived from analyses of data collected in the PPMI observational clinical study. Details regarding the PPMI study have been previously published and are available at ppmi-info.org $(52,54)$. Patients from the PPMI population were selected for comparison with the PASADENA cohort using the following criteria, which align with the PASADENA study criteria: to have at least two of the following: resting tremor, bradykinesia, rigidity (must have either resting tremor or bradykinesia), and confirmation from imaging core that DaTSPECT screening was consistent with dopamine transporter deficit. Data were downloaded in May 2020 and, from the total 423 individuals in the PPMI population, a cohort of 336 participants were selected based on the above criteria. No individuals in the PPMI cohort received MAO-B inhibitors at baseline.

\section{Statistical Comparisons Between PASADENA and PPMI Cohorts}

The standardized mean difference (SMD) in prognostic scores was used to assess differences between baseline data for the PASADENA and PPMI cohorts, and for the PASADENA MAO$B$ inhibitor-treated vs. treatment-naïve patient groups. The SMD was calculated as the absolute value in the difference in means of a covariate across the treatment groups, divided by the 
pooled standard deviation (SD). SMDs larger than 0.25 indicate that the groups were too different from one another for a reliable comparison of change from baseline in that variable (55). Overall, 20 covariates were selected for the analysis, including demographic, imaging, and clinical assessment (MDS-UPDRS Parts I + II + III) data.

\section{RESULTS OF BASELINE DATA ANALYSIS Baseline PASADENA Demographics}

A total of 443 patients were screened between June 2017 and November 2018 at 60 sites. Overall, 127 patients failed screening due to not meeting certain inclusion and exclusion criteria, such as brain DaT-SPECT screening consistent with $\mathrm{PD}$, concomitant disease, or condition within 6 months of screening and MMSE $\leq 25$. Overall, 316 patients were enrolled at 57 centers across the following five countries: United States (160 patients [50.6\%]), Spain (50 patients [15.8\%]), France (65 patients [20.6\%]), Germany (35 patients [11.1\%]), and Austria (6 patients $[1.9 \%])$. Of those patients enrolled into the PASADENA study, 115 (36\%) had received MAO-B inhibitor treatment at enrollment and 201 (64\%) were treatment naïve. The mean (SD) age of PASADENA patients was 59.9 (9.10) years and the population included $213(67.4 \%)$ men and 103 (32.6\%) women, with a mean time from diagnosis of 10.11 (6.50) months and 238 (75.3\%) individuals being H\&Y Stage II. The mean (SD) MDSUPDRS sum of Parts I + II + III at baseline was 31.41 (12.78) and the mean total scores for the individual parts were: Part I, 4.61 (3.83); Part II, 5.33 (4.04); and Part III, 21.47 (9.00). The mean baseline DaT-SPECT striatal binding ratios for the PASADENA population were $0.80(0.25)$ for the contralateral putamen and $1.06(0.32)$ for the ipsilateral putamen.

\section{MAO-B Inhibitor-Treated vs. Treatment-Naïve Patients in the PASADENA Population}

Within the PASADENA study population, patients treated with MAO-B inhibitors at baseline were on average younger (58.2 [9.00] years vs. 60.8 [9.00] years, respectively; SMD:-0.290) and had a longer time from diagnosis (11.96 [6.10] months vs. 9.06 [6.50] months, respectively; SMD: 0.461) vs. the treatmentnaïve group. MAO-B inhibitor-treated patients also had a higher MoCA score vs. the treatment-naïve group (28.27 [1.96] vs. 27.65 [2.04], respectively; SMD: 0.309). All other baseline characteristics were balanced between patients who received a MAO-B inhibitor $(n=115)$ and those who were treatment naïve ( $n=201$; Table 1 and Supplementary Figure 1 ).

\section{PASADENA Population vs. PPMI Cohort, Selected Using PASADENA Eligibility Criteria}

When comparing the PASADENA population baseline characteristics with those of the PPMI cohort, MDS-UPDRS mean scores were lower for the PASADENA population for Part I (4.61 [3.83] vs. 5.60 [3.93], respectively; SMD: 0.255) and Part IB (3.45 [2.78] vs. 4.33 [3.10], respectively; SMD: 0.297) compared with the PPMI cohort. The PASADENA population had longer average time from diagnosis (10.11 [6.50] months vs. 6.44 [6.31] months, respectively; SMD: -0.573) compared with the PPMI cohort. DaT-SPECT striatal binding ratios for the PASADENA population for the contralateral putamen $(0.80$ [0.25] vs. 0.68 [0.27], respectively; SMD: -0.445) and ipsilateral putamen (1.06 [0.32] vs. 0.96 [0.39], respectively; SMD: -0.285 ) to the clinically most affected side were both higher compared with the PPMI cohort. On average, PASADENA patients scored higher in MoCA (27.87 [2.03] vs. 27.24 [2.29], respectively; SMD: -0.291) and lower in SCOPA-AUT (8.05 [5.71] vs. 9.75 [6.23], respectively; SMD: 0.284) scores compared with the PPMI cohort. All other baseline characteristics were balanced between the PASADENA population and the PPMI cohort (Table 1 and Supplementary Figure 2).

\section{DISCUSSION}

PASADENA is the first Phase II study to test the efficacy of a monoclonal antibody that selectively binds aggregated alpha-synuclein to slow disease progression in early PD. The PASADENA study enrolled individuals diagnosed with earlystage $\mathrm{PD}$, requiring that they be, inter alia, either treatment naïve or on stable treatment with MAO-B inhibitors and have a DaTSPECT-confirmed dopaminergic deficit. In order for this Phase II proof-of-concept study to measure the disease-modifying potential of prasinezumab within the study period of 1 year, it was essential to define a population with a measurable rate of progression. The progression rate may depend on baseline disease severity and other factors (56). Several previous studies have reported that the progression rate in individuals with early PD is generally faster shortly after diagnosis of PD and before the start of levodopa or dopamine agonist therapy usage $(47,57)$. Individuals at this early stage of disease still have vulnerable dopaminergic neurons and protecting them against the development of further alpha-synuclein pathology may potentially slow motor disease progression. A 1-year treatment duration, if well-powered, is expected to be sufficient to demonstrate relevant between-group differences, resulting from an effect of treatment on disease progression in individuals with early PD.

Current treatments for PD, such as levodopa, improve motor symptoms and aim to increase dopamine levels, compensating for the dopaminergic cell and synaptic loss (58). It is possible that the potential effects of treatment with a disease-modifying therapy on motor symptoms in individuals with early PD might be masked by these powerful symptomatic therapies $(47,52)$. People with early PD treated with MAO-B inhibitors have a reduced likelihood of starting levodopa or dopamine agonists therapy compared with placebo, while maintaining a relatively high progression rate $(56,57)$. Individuals with early PD who were either treatment naïve or treated with a MAO-B inhibitor at baseline were, therefore, included in Part 1 of the PASADENA study. The recruited PASADENA study population was also similar to other early PD therapeutic trial populations $(56,59,60)$. 
TABLE 1 | PASADENA; MAO-B inhibitor-treated vs. treatment-naïve patients, and PASADENA (all patients) vs. PPMI.

\begin{tabular}{|c|c|c|c|c|c|c|}
\hline & $\begin{array}{c}\text { PASADENA } \\
\text { MAO-B } \\
\text { inhibitor-treated } \\
\text { patients } \\
(n=115)\end{array}$ & $\begin{array}{c}\text { PASADENA } \\
\text { Treatment-naïve } \\
\text { patients } \\
(n=201)\end{array}$ & SMD (CI) & $\begin{array}{c}\text { PASADENA } \\
\text { All patients }(n= \\
316)\end{array}$ & $\begin{array}{c}\text { PPMI } \\
\text { PD patients }(n= \\
336)\end{array}$ & SMD (CI) \\
\hline Age, years mean (SD) & $58.2(9.00)$ & $60.8(9.00)$ & $-0.290^{*}(-0.520,-0.059)$ & $59.90(9.10)$ & $61.30(9.69)$ & $0.166(0.012,0.320)$ \\
\hline Gender Male, $n(\%)^{\dagger}$ & $74(64.3)$ & $139(69.2)$ & $-0.048(-0.156,0.060)$ & $213(67.4)$ & $220(65.5)$ & $-0.019(-0.092,0.053)$ \\
\hline Years of education, mean (SD) & $16.39(5.20)$ & $15.22(4.80)$ & $0.234(0.006,0.467)$ & $15.65(4.99)$ & $15.53(3.03)$ & $-0.029(-0.183,0.125)$ \\
\hline Time from diagnosis, months mean (SD) & $11.96(6.10)$ & $9.06(6.50)$ & $0.461^{\star}(0.225,0.689)$ & $10.11(6.50)$ & $6.44(6.30)$ & $-0.573^{\star}(-0.730,-0.417)$ \\
\hline H\&Y Stage II, $n(\%)^{\dagger}$ & $83(72.2)$ & $155(77.1)$ & $-0.049(-0.150,0.051)$ & $238(75.3)$ & $197(59.0)$ & $-0.167(-0.238,-0.096)$ \\
\hline RBDSQ (SD) & $3.51(2.65)$ & $3.43(2.75)$ & $0.031(-0.199,0.260)$ & $3.46(2.71)$ & $4.14(2.69)$ & $0.250(0.095,0.405)$ \\
\hline DaT-SPECT contralateral putamen (SD) & $0.81(0.24)$ & $0.78(0.25)$ & $-0.102(-0.331,0.128)$ & $0.80(0.25)$ & $0.68(0.27)$ & $-0.445^{\star}(-0.601,-0.289)$ \\
\hline DaT-SPECT ipsilateral putamen (SD) & $1.02(0.30)$ & $1.09(0.33)$ & $-0.194(-0.421,0.038)$ & $1.06(0.32)$ & $0.96(0.39)$ & $-0.285^{*}(-0.439,-0.129)$ \\
\hline MoCA (SD) & $28.27(1.96)$ & $27.65(2.04)$ & $0.309^{\star}(0.076,0.539)$ & $27.87(2.03)$ & $27.24(2.29)$ & $-0.291^{*}(-0.446,-0.136)$ \\
\hline SCOPA-AUT (SD) & $7.71(4.82)$ & $8.25(6.16)$ & $-0.096(-0.323,0.136)$ & $8.05(5.71)$ & $9.75(6.23)$ & $0.284^{\star}(0.128,0.439)$ \\
\hline MDS-UPDRS sum of Parts I + II + III, mean (SD) & $30.21(11.96)$ & $32.10(13.20)$ & $-0.150(-0.378,0.081)$ & $31.41(12.78)$ & $32.63(13.04)$ & $0.094(-0.059,0.248)$ \\
\hline MDS-UPDRS Part I, mean (SD) & $4.49(3.40)$ & $4.68(4.06)$ & $-0.051(-0.279,0.180)$ & $4.61(3.83)$ & $5.60(3.93)$ & $0.255^{\star}(0.100,0.409)$ \\
\hline MDS-UPDRS Part IA, mean (SD) & $0.96(1.54)$ & $1.27(1.67)$ & $-0.195(-0.422,0.037)$ & $1.16(1.62)$ & $1.27(1.57)$ & $0.071(-0.083,0.224)$ \\
\hline MDS-UPDRS Part IB, mean (SD) & $3.53(2.50)$ & $3.14(2.93)$ & $0.045(-0.185,0.273)$ & $3.45(2.78)$ & $4.33(3.10)$ & $0.297^{\star}(0.142,0.451)$ \\
\hline MDS-UPDRS Part II, mean (SD) & $5.19(3.90)$ & $5.41(4.13)$ & $-0.055(-0.284,0.174)$ & $5.33(4.04)$ & $6.12(4.20)$ & $0.190(0.036,0.344)$ \\
\hline MDS-UPDRS Part III, mean (SD) & $20.53(8.81)$ & $22.01(9.09)$ & $-0.165(-0.394,0.065)$ & $21.47(9.00)$ & $20.92(8.88)$ & $-0.062(-0.216,0.092)$ \\
\hline MDS-UPDRS Part III Axial Symptoms, mean (SD) ${ }^{\dagger}$ & $0.77(0.50)$ & $0.78(0.57)$ & $-0.004(-0.233,0.225)$ & $0.78(0.54)$ & $0.68(0.71)$ & $-0.153(-0.307,0.001)$ \\
\hline MDS-UPDRS Part III Bradykinesia, mean (SD) $)^{\ddagger}$ & $9.91(5.60)$ & $10.48(5.49)$ & $-0.102(-0.331,0.127)$ & $10.27(5.53)$ & $10.60(5.60)$ & $0.059(-0.095,0.212)$ \\
\hline MDS-UPDRS Part III Rigidity, mean (SD) $\left.\right|^{\ddagger}$ & $3.90(2.58)$ & $4.17(2.83)$ & $-0.103(-0.331,0.128)$ & $4.07(2.74)$ & $3.86(2.61)$ & $-0.078(-0.232,0.075)$ \\
\hline MDS-UPDRS Part III Resting Tremors, mean (SD) ${ }^{\ddagger}$ & $2.71(2.77)$ & $3.12(2.74)$ & $-0.147(-0.377,0.082)$ & $2.97(2.75)$ & $2.58(2.42)$ & $-0.151(-0.305,0.003)$ \\
\hline
\end{tabular}

Demographics and baseline disease characteristics compared using the SMD. ${ }^{*}$ Indicates not balanced covariates (>0.25 SMD). ${ }^{\dagger}$ For binary variables the table shows the difference in proportions. ${ }^{\star}$ Part III subscores are defined as: Bradykinesia (sum of item 3.4, finger tapping; item 3.5, hand movements; item 3.6, pronation-supination movements of hands; item 3.7, toe tapping; item 3.8, leg agility; item 3.9, arising from chair; ;tem 3.13, posture; and item 3.14, body bradykinesia); Rigidity (sum of item 3.3. [Neck, Upper Limbs and Lower Limbs]); Resting tremors (sum of item 3.17, rest tremor amplitude [Lip/Jaw, Upper Limbs and Lower Limbs] and Item 3.18, constancy of tremor); and axial symptoms (sum of item 3.10, gait; item 3.11, freezing of gait; and item 3.12, postural stability). Cl, confidence interval; DaT-SPECT, dopamine transporter with single-photon emission computerized tomography; H\&Y, Hoehn and Yahr; MAO-B, monoamine oxidase B; MDS-UPDRS, Movement Disorders Society - Unified Parkinson's Disease Rating Scale; MoCA, Montreal Cognitive Assessment: PD, Parkinson's disease; PPMI, Parkinson's Progression Markers Initiative; RBDSQ, Rapid Eye Movement Sleep Behavior Disorder Screening Questionnaire; SCOPA-AUT, Scales for Outcomes in Parkinson's Disease-Autonomic Dysfunction; SD, standard deviation; SMD, standardized mean difference. 
In this study, a comparison of the baseline characteristics of PASADENA study participants showed that those who received treatment with MAO-B inhibitors were younger with a longer time from diagnosis compared with the treatment-naïve group; however, the two groups had similar overall symptom severity at baseline. This observed similarity may be due to improvement in MDS-UPDRS scores as a result of MAO-B inhibitor treatment, which provides symptomatic relief to individuals with early PD by prolonging the action of dopamine in the brain $(61,62)$. The younger age may be due to the fact that individuals with older onset of PD usually receive levodopa rather than MAO-B inhibitors (63).

The design and assumptions of this study were informed from analyses of data collected in the PPMI observational clinical study. Therefore, it was important to compare the baseline characteristics of the PASADENA study population with the PPMI cohort to ensure the assumptions made were also valid for the PASADENA population. The PASADENA population and PPMI cohort showed different distributions for some demographic measures; for example, more PASADENA participants were in H\&Y Stage II (75 vs. 59\%) and had on average a 3.7-month-longer time from diagnosis than the PPMI cohort. However, the DaT-SPECT striatal binding ratios for both the ipsilateral and contralateral putamen suggested that the PASADENA population had slightly less advanced disease in terms of severity and potentially of progression.

Dose selection for the PASADENA trial was based on data from previous studies. In a Phase I multiple-ascending-dose study (NCT02157714), individuals with mild-to-moderate PD who received prasinezumab up to $60 \mathrm{mg} / \mathrm{kg}$ intravenously every 4 weeks reported no serious or severe adverse events (11). Rapid-dose and time-dependent mean reductions from baseline vs. placebo in free serum alpha-synuclein levels of up to $97 \%$ were reported in trial participants after a single infusion at the highest dose $(P=0.002)$, with similar reductions after two additional infusions. Mean cerebrospinal fluid concentration of prasinezumab also increased with dose, to $\sim 0.3 \%$ relative to the concentration in serum across all dose cohorts. Currently, assays to quantify engagement of prasinezumab with aggregated forms of alpha-synuclein in vivo are not available. Thus, the dose selection for the Phase II study was primarily based on human serum and cerebrospinal fluid PK data extrapolation, and the relationship of these data with histopathological and functional endpoints. The doses used in the PASADENA trial were selected to fall in the therapeutic exposure range predicted from preclinical efficacy models; the high prasinezumab dose (4,500 mg for body weight $\geq 65 \mathrm{~kg} ; 3,500 \mathrm{mg}$ for body weight $<65 \mathrm{~kg}$ ) was selected to match exposure at the $60 \mathrm{mg} / \mathrm{kg}$ dose in the multiple-ascending-dose study, and the $1,500 \mathrm{mg}$ prasinezumab dose to yield exposure levels above those effective on alpha-synuclein pathology in the mouse model, with sufficient separation between the two to enable exposure response analyses. Both doses selected were expected to bind $>90 \%$ of pathological aggregated alpha-synuclein in the central nervous system, thus both could show signal of efficacy on disease progression (11).

The effect of treatment with prasinezumab on clinical progression rate was determined using the MDS-UPDRS and was supported with a panel of exploratory biomarkers assessing the potential effects on PD pathology and progression of neuronal damage. The MDS-UPDRS is comprised of four parts: Part I, Mentation, Behavior, and Mood; Part II, Activities of Daily Living; Part III, Motor Examination; and Part IV, Complications of Therapy $(49,64)$. Each parkinsonian sign or symptom is rated on a 5-point scale (ranging from 0 to 4), with higher scores indicating more severe impairment (64). The MDSUPDRS demonstrates good reliability, validity and sensitivity to change over a range of measures of time from diagnosis and severity (64). Previous studies in individuals with early PD have demonstrated a linear increase in MDS-UPDRS of $\sim 6-12$ points per year following diagnosis and prior to initiating symptomatic treatment $(47,52,56,57)$. A positive effect on MDS-UPDRS scores may, therefore, indicate a potential effect of prasinezumab on global PD progression. It is important to note that the increase in MDS-UPDRS scores in the treatment-naïve population with early PD is derived from the Part III motor examination scores; this population exhibits decline to a far lesser extent in activities of daily living (MDS-UPDRS Part I) and motor problems in daily life (MDS-UPDRS Part II) (64).

A deficit of striatal dopamine transporters, measured by DaTSPECT imaging, is used to confirm that neurodegeneration of striatal dopaminergic terminals is indicative of PD (65). Individuals at the earliest stage of $\mathrm{PD}$ show the fastest inverse exponential decline of DaT-SPECT signal (i.e., loss of dopaminergic terminals) in the ipsilateral putamen to the most clinically affected side $(66,67)$. Therefore, DaT-SPECT in the ipsilateral putamen was selected as a secondary outcome measure in the PASADENA study to determine the disease-modifying potential of prasinezumab on the neurodegeneration of the nigrostriatal terminals.

Smartphones and smartwatches are built with high-quality sensors that, together with novel software technologies, enable the remote, non-invasive, frequent, and sensitive measurement and analysis of motor symptoms in PD (68-70). Digital monitoring of motor symptoms using smartphones has been previously used in the Phase I study of prasinezumab in individuals with PD (NCT02157714), in which preliminary reliability and clinical validity were established (71). Study participants completed a daily battery of tests and carried the phone with them throughout the day for passive monitoring. The study revealed high adherence and a strong correlation between smartphone sensor data and clinical measures of motor signs and, notably, the detection of clinical manifestations that were not apparent at site visits (71). A second version of this digital biomarker approach was therefore implemented in the PASADENA study to maximize the probability of detecting a potential therapeutic effect of prasinezumab and potentially provide new insights into the functioning and behavior of individuals with PD. The test-retest reliability and preliminary clinical validity of the sensor-based outcome measures were demonstrated with high intraclass correlation coefficients and significant correlations with MDS-UPDRS Part III subscale items, respectively (72). As mentioned, summary digital scores should be developed independent of clinical measures. However, the independent longitudinal sensor datasets 
required to build and validate such a digital score are not yet available (50). Therefore, the "PASADENA Digital Motor Score" was developed as an exploratory outcome measure for this study to reflect global motor function. We note that this exploratory outcome measure would require validation in an independent cohort before elevating this measure higher in the endpoint hierarchy.

A definitive diagnosis of PD can only be made post-mortem as biomarker tools that detect alpha-synuclein in the brain in vivo are not currently available (73). However, pathological forms of alpha-synuclein have been detected in peripheral neurons present in skin biopsy samples from individuals with PD; the degree of peripheral nerve pathology detected in samples was found to correlate with disease severity $(74,75)$. Longitudinal skin biopsy sampling has been implemented in the PASADENA study for the direct and in vivo assessment of alpha-synuclein pathology, and its progression in response to treatment with prasinezumab. Clinical data and samples, including skin biopsies, will be collected from DaT-SPECT negative screen failure participants to determine whether the detection of alpha-synuclein skin pathology may be used as a sensitive, specific, and less invasive tool to diagnose PD.

While this study was designed to assess the safety and efficacy of prasinezumab in early PD, there were some limitations to the study. In order to assess the disease-modifying effect of a drug in $\mathrm{PD}$, the disease must progress by a measurable amount during the study period. Over a 1-year period, individuals with early PD progress $<2$ points on both MDS-UPDRS Parts I and II (47). This is below the threshold for clinical meaningfulness ( $>2$ points) when a patient's quality of life is negatively affected (76). However, the MDS-UPDRS Part III progresses more quickly in early $\mathrm{PD}$ and can often pass the threshold for clinical meaningfulness; 4.63 points $(47,76)$. This may in part be due to the original UPDRS being developed when PD was thought to be a predominantly motor disease, thus, non-motor symptoms such as constipation, fatigue and sleep problems are inadequately represented on the scale (77).

This study included patient-reported outcome measures of quality of life (e.g., PGI-C, SE-ADL, CGI-I); however, the change in these outcome measures is usually minimal in early PD. Therefore, there is a need for a sensitive outcome measure that captures both early and non-motor PD symptoms, addresses symptom diversity and is correlated with clinically meaningful impacts on quality of life $(53,77)$. Another limitation of studies assessing disease progression in $\mathrm{PD}$ is the confounding effect of dopaminergic PD treatment. We accounted for this by analyzing the change in MDS-UPDRS using a hypothetical estimand strategy, in which the participants are included in the model until the visit prior to starting dopaminergic PD treatment. In addition, we performed a sensitivity analysis using the treatment policy estimand strategy, in which all participants are included regardless of starting dopaminergic PD treatment. In this analysis, we have tried to account for the confounding effect of dopaminergic PD treatment by including an 8-h off medication time for the "Off" evaluation in this study, the long duration response to levodopa may begin to have a PK impact on patients within their first years of levodopa use (78). Ideally, we would recruit untreated individuals in the prodromal stage of PD, however, there are currently no available biomarkers that give a definitive diagnosis of prodromal PD (79). Therefore, we included participants with a recent clinical diagnosis of PD $(<2$ years), as this is currently the earliest stage at which PD can be definitively identified.

Finally, proof-of-concept studies can present statistical challenges, such as smaller sample sizes, which can reduce statistical power. In this study, the Sponsor decided to use an $80 \%$ confidence interval $(\alpha=0.20)$ rather than a more common $95 \%(\alpha=0.05)$ to ensure completion of the trial in an acceptable timeframe. Although reducing the sample size may lead to less precision, point estimates reported together with $80 \%$ confidence intervals still provide useful information about the plausible magnitude of the effect between prasinezumab and placebo, as lower confidence levels decrease the width of the confidence interval. The trade-off between sample size and confidence level confirmed that the endpoints with a confidence interval that does not contain the value of null effect should be investigated in future clinical trials (80).

\section{CONCLUSIONS}

The PASADENA Phase II study was designed to assess the safety and tolerability and clinical effect of prasinezumab on disease progression in patients with early PD. This study will focus on the effect of treatment in early $\mathrm{PD}$, as disease progression is measurable and predictable in this cohort and will not be masked by treatment with dopaminergic therapy. The primary outcome measure will be supported by clinical measures and imaging to investigate the potential physiological impact of treatment. In addition, novel digital biomarkers will be used to assess potentially subtle effects of treatment on motor function in individuals with PD.

\section{DATA AVAILABILITY STATEMENT}

The datasets presented in this study can be found in online repositories. Qualified researchers may request access to individual patient-level data through the clinical study data request platform (https://vivli.org/). Further details on Roche's criteria for eligible studies are available here (https:/vivli.org/ members/ourmembers/). For further details on Roche's Global Policy on the Sharing of Clinical Information and how to request access to related clinical study documents, see here (https://www. roche.com/research_and_development/who_we_are_how_we_ work/clinical_trials/our_commitment_to_data_sharing.htm). Data used in the preparation of this article were obtained from the Parkinson's Progression Markers Initiative (PPMI) database (ppmi-info.org/data). For up-to-date information on the study, visit ppmi-info.org.

\section{ETHICS STATEMENT}

The studies involving human participants were reviewed and approved by Ethikkommission der Medizinischen 
Universität Innsbruck, Innsbruck, Austria; Comité de Protection des Personnes (CPP) Ouest IV, Nantes, France; Ethikkommission der Universität Leipzig and Geschäftsstelle der Ethikkommission an der medizinischen Fakultät der Universität Leipzig, Leipzig, Germany; Ethikkommission der Fakultät für Medizin der Technischen Universität München, München, Germany; Ethikkommission der Universität Ulm (Oberer Eselsberg), Ulm, Germany; Landesamt für Gesundheit und Soziales Berlin and Geschäftsstelle der Ethik-Kommission des Landes Berlin, Berlin, Germany; Ethikkommission des FB Medizin der Philipps-Universität Marburg, Marburg, Germany; Ethikkommission an der Medizinischen Fakultät der Eberhard-Karls-Universität und am Universitätsklinikum Tübingen, Tübingen, Germany; Ethikkommission an der Med. Fakultät der HHU Düsseldorf, Düsseldorf, Germany; Ethikkommission der LÄK Hessen, Frankfurt, Germany; CEIm Hospital Universitari Vall d'Hebron, Barcelona, Spain; Copernicus Group Independent Review Board, Puyallup, Washington, USA; Western Institutional Review Board, Puyallup, Washington, USA; The University of Kansas Medical Center Human Research Protection Program, Kansas City, Kansas, USA; Oregon Health \& Science University Independent Review Board, Portland, Oregon, USA; Northwestern University Institutional Review Board, Chicago, Illinois, USA; Spectrum Health Human Research Protection Program, Grand Rapids, Michigan, USA; The University of Vermont Committees on Human Subjects, Burlington, Vermont, USA; Beth Israel Deaconess Medical Center Committee on Clinical Investigations, New Procedures and New Forms of Therapy, Boston, Massachusetts, USA; Vanderbilt Human Research Protection Program Health, Boston, Massachusetts, USA; Vanderbilt Human Research Protection Program Health, Nashville, Tennessee, USA; University of Maryland, Baltimore Institutional Review Board, Baltimore, Maryland, USA; University of Southern California Institutional Review Board, Los Angeles, California, USA; Columbia University Medical Center Institutional Review Board, New York, New York, USA; University of Southern California San Francisco Institutional Review Board, San Francisco, California, USA; University of Pennsylvania Institutional Review Board, Philadelphia, Philadelphia, USA; HCA-HealthOne Institutional Review Board, Denver, Colorado, USA. The patients/participants provided their written informed consent to participate in this study.

\section{PASADENA INVESTIGATORS}

Claudia Altendorf, Chareyna Anandan, Giulia Andrews, Solène Ansquer, Raphaele Arrouasse, Sana Aslam, JeanPhilippe Azulay, Jeanette Baker, Ernest Balaguer Martinez, Shadi Barbu, Kara Bardram, Danny Bega, Helena Bejr-Kasem Marco, Isabelle Benatru, Eve Benchetrit, Felix Bernhard, Amir Besharat, Sagari Bette, Amelie Bichon, Andrew Billnitzer, Sophie Blondeau, Thomas Boraud, Freiderike Borngräber, James Boyd, Kathrin Brockmann, Matthew Brodsky, Ethan Brown, Christof Bruecke, Fabienne Calvas, Monica Canelo, Federico
Carbone, Claire Carroll, Laura Casado Fernandez, Catherine Cassé-Perrot, Anna Castrioto, Helene Catala, Justine Chan, Samia Cheriet, Anthony Ciabarra, Joseph Classen, Juliana Coleman, Robert Coleman, Yaroslau Compta, Anne-Gaëlle Corbillé, Jean-Christophe Corvol, Mariana Cosgaya, Nabila Dahodwala, Philippe Damier, Elodie David, Thomas Davis, Marissa Dean, Berengere Debilly, Janell DeGiorgio, Andres Deik, Laure Delaby, Marie-Helene Delfini, Pascal Derkinderen, Philipp Derost, Maria de Toledo, Lisa Deuel, Ann Marie Diaz-Hernandez, Cameron Dietiker, Karina Dimenshteyn, Julio Dotor, Franck Durif, Jens Ebentheuer, Karla Maria Eggert, Sara Eichau Madueño, Claudia Eickhoff, Aaron Ellenbogen, Philipp Ellmerer, Ines Esparragosa Vazquez, Alexandre Eusebio, Siobhan Ewert, John Fang, Danielle Feigenbaum, Frederique Fluchere, Alexandra Foubert-Samier, Marie Fournier, Anne Fradet, Valerie Fraix, Samuel Frank, Franca Fries, Monique Galitzky, Marisol Gallardó Pérez, Jose Manuel García Moreno, Carmen Gasca, Thomas Gasser, Joyce Gibbons, Caroline Giordana, Alicia Gonzalez Martinez, Ira Goodman, Arantza Gorospe, Marie Goubeaud, David Grabli, Mangone Graziella, Stephan Grimaldi, Jeffrey Gross, Raquel Guimaraes-Costa, Andreas Hartmann, Christian Hartmann, Travis Hassell, Robert Hauser, Antonio Hernandez, Jorge Hernandez-Vara, Günter Höglinger, Christian Homedes, Andrea Horta-Barba, Jean-Luc Houeto, Julius Huebl, Jennifer Hui, Stuart Isaacson, Joseph Jankovic, Annette Janzen, Junior Jauregui, Jocelyne Jiao, Maria Jose Marti Domenech, Xavier Joseph, Srinath Kadimi, Pat Kaminski, Silja Kannenberg, Jan Kassubek, Maya Katz, Kevin Klos, Shannon Klos, Christopher Kobet, Jennifer Koebert, Patricia Krause, Andrea Kuehn, Jaime Kulisevsky Bojarsky, Rajeev Kumar, Martin Kunz, Lille Kurvits, Kimberly Kwei, Simon Laganiere, Brice Laurens, Johannes Levin, Oren Levy, Peter LeWitt, Gurutz Linazasoro Cristóbal, Irene Litvan, Karlo Lizarraga, Katherine Longardner, Rocio Lopez, Lydia Lopez Manzanares, Sara Lucas del Pozo, Maria Rosario Luquin Puido, Nijee Luthra, Kelly Lyons, Sylvia Maass, Gerrit Machetanz, Yolanda Macias, David Maltete, Jorge Uriel Manez Miro, LouiseLaure Mariani, Juan Marin, Kathrin Marini, Ana Marques, Gloria Marti, Maria Jose Marti Domenech, Saul Martinez, Wassilios Meissner, Sara Meoni, Brit Mollenhauer, Dunia Mon Martinez, Johnson Moon, Elena Moro, Peter Morrison, Christoph Muehlberg, Manpreet Multani, Christine Murphy, Anthony Nicholas, Rajesh Pahwa, Antonio Palasi, Heidi Pape, Neepa Patel, Prity Patel, Marina Peball, Elizabeth Peckham, Terry Peery, Rafael Perez, Jesus Perez, Alisa Petit, Elmar Pinkhardt, Werner Poewe, Elsa Pomies, Cecile Preterre, Joseph Quinn, Olivier Rascol, Philippe Remy, Irene Richard, Benjamin Roeben, Emily Ruether, Jost-Julian Rumpf, David Russell, Hayet Salhi, Daniela Samaniego-Toro, Alexandra Samier-Foubert, Antonio Sanchez, Emmanuelle Schmitt, Alfons Schnitzler, Oliver Schorr, Julie Schwartzbard, Kerstin Schweyer, Klaus Seppi, Victoria Sergo, Holly Shill, Andrew Siderowf, Tanya Simuni, Umberto Spampinato, Ashok Sriram, Natividad Stover, Caroline Tanner, Arjun Tarakad, Carolyn Taylor, Claire Thalamus, Thomas Toothaker, Nadege Van Blercom, Nora VanegasArrogave, Lydia Vela, Sylvian Vergnet, Tiphaine Vidal, Jonathan Vöglein, Ryan Walsh, Cheryl Waters, Mirko Wegscheider, Endy 
Weidinger, Caroline Weill, Gregor Wenzel, Tatiana Witjas, Isabel Wurster, Brenton Wright, Milan Zimmermann, and Rafael Zuzuarregui.

\section{PRASINEZUMAB STUDY GROUP}

Markus Abt, Atieh Bamdadian, Teresa Barata, Nicholas Barbet, Sara Belli, Frank Boess, Azad Bonni, Edilio Borroni, Anne Boulay, Markus Britschgi, Jerome Chague, Valerie Cosson, Christian Czech, Dennis Deptula, Cheikh Diack, Rachelle Doody, Juergen Dukart, Giulia D'Urso, Sebastian Dziadek, Hannah Eddleston, Chris Edgar, Laurent Essioux, Morgan Farell, Rebecca Finch, Paulo Fontoura, Waltraud Gruenbauer, Andrea Hahn, Stefan Holiga, Michael Honer, Shirin Jadidi, Kelly Johnson-Wood, Markus Keller, Timothy Kilchenmann, Martin Koller, Thomas Kremer, Thomas Kustermann, Claire Landsdall, Michael Lindemann, Florian Lipsmeier, Cecile Luzy, Marianne Manchester, Maddalena Marchesi, Ferenc Martenyi, Meret Martin-Facklam, Katerina Mironova, Annabelle Monnet, Emma Moore, Daniel K Ness, Markus Niggli, Tania Nikolcheva, Susanne Ostrowitzki, Gennaro Pagano, Benedicte Passmard, Agnes Poirier, Anke Post, Megana Prasad, Nathalie Pross, Tiffany Quock, Benedicte Ricci, Ellen Rose, Christoph Sarry, Christine Schubert, Dennis Selkoe, Jeff Sevigny, Kaycee Sink, Hannah Staunton, Tim Steven, Alexander Strasak, Hanno Svoboda, Kirsten Taylor, Radhika Tripuraneni, Dylan Trundell, Daniel Umbricht, Lynne Verselis, Annamarie Vogt, Ekaterina Volkova-Volkmar, Cornelia Weber, Silke Weber, and Wagner Zago.

\section{AUTHOR CONTRIBUTIONS}

GP, FB, KT, BM, WP, GK, WZ, DN, SO, KM, MK, and JS designed the study. GP, FB, KT, BM, WP, ABou, AV, MM, $\mathrm{AP}, \mathrm{SO}$, and TS were involved in data collection. GP, KT, $\mathrm{BR}$, JA-C, $\mathrm{AV}$, and $\mathrm{MM}$ analyzed the data. GP and ABon drafted the work. GP, FB, KT, BR, BM, WP, JA-C, AP, TN, GK, WZ, HS, SO, TS, KM, MK, JS, RD, PF, DU, and ABon

\section{REFERENCES}

1. Verschuur CVM, Suwijn SR, Boel JA, Post B, Bloem BR, van Hilten JJ, et al. Randomized delayed-start trial of levodopa in Parkinson's disease. New Engl J Med. (2019) 380:315-24. doi: 10.1056/NEJMoa1809983

2. Lang AE, Espay AJ. Disease modification in Parkinson's disease: current approaches, challenges, and future considerations. Mov Disord. (2018) 33:660-77. doi: $10.1002 / \mathrm{mds} .27360$

3. Cheng HC, Ulane CM, Burke RE. Clinical progression in Parkinson disease and the neurobiology of axons. Ann Neurol. (2010) 67:715-25. doi: 10.1002/ana.21995

4. Jankovic J, Aguilar LG. Current approaches to the treatment of Parkinson's disease. Neuropsychiatr Dis Treat. (2008) 4:743-57. doi: 10.2147/NDT.S2006

5. Bonnet AM. Involvement of non-dopaminergic pathways in Parkinson's disease. CNS Drugs. (2000) 13:351-64. doi: 10.2165/00023210-200013050-00005 critically revised important intellectual content. GP and ABon provided final approval of the manuscript. All authors were involved in data interpretation and revised and gave input on the article.

\section{FUNDING}

The authors declare that F. Hoffmann-La Roche Ltd was the sponsor and sole funder of the study.

\section{ACKNOWLEDGMENTS}

The authors thank all the subjects who participated in this study. PPMI was a public-private partnership sponsored by the Michael J. Fox Foundation (MJFF) for Parkinson's Research and is co-funded by MJFF, AbbVie, Allergan, Amathus Therapeutics, Avid Radiopharmaceuticals, Bial Biotech, Biogen Idec, BioLegend, Bristol-Myers Squibb, Calico, Celgene, Denali Therapeutics Inc., 4D Pharma Plc, Eli Lilly and Company, F. Hoffmann-La Roche Ltd., GE Healthcare, Genentech Inc., GlaxoSmithKline, Golub Capital BDC, Handl Therapeutics, Insitro, Janssen Neuroscience, Lundbeck, Merck, Meso Scale, Neurocrine Biosciences, Pfizer, Piramal, Prevail Therapeutics, Sanofi Genzyme, Servier, Takeda, Teva Pharmaceutical Industries Ltd., UCB, Verily Life Sciences, and Voyager Therapeutics. Industry partners contribute to PPMI through financial and in-kind donations and have a lead role in providing feedback on study parameters through the Partners Scientific Advisory Board (PSAB). Through close interaction with the study, the PSAB was positioned to inform the selection and review of potential progression markers that could be used in clinical testing.

\section{SUPPLEMENTARY MATERIAL}

The Supplementary Material for this article can be found online at: https://www.frontiersin.org/articles/10.3389/fneur. 2021.705407/full\#supplementary-material
6. Giguere N, Burke Nanni S, Trudeau LE. On cell loss and selective vulnerability of neuronal populations in Parkinson's disease. Front Neurol. (2018) 9:455. doi: $10.3389 /$ fneur.2018.00455

7. Lees AJ. The on-off phenomenon. J Neurol Neurosurg Psychiatry. (1989) 52(Suppl.) 29-37. doi: 10.1136/jnnp.52.Suppl.29

8. Jenner P. Treatment of the later stages of Parkinson's disease pharmacological approaches now and in the future. Transl Neurodegener. (2015) 4:3. doi: 10.1186/2047-9158-4-3

9. Martinez-Martin P, Rodriguez-Blazquez C, Forjaz MJ. Quality of life and burden in caregivers for patients with Parkinson's disease: concepts, assessment and related factors. Expert Rev Pharmacoeconomics Outcomes Res. (2012) 12:221-30. doi: 10.1586/erp.11.106

10. Seppi K, Ray Chaudhuri K, Coelho M, Fox SH, Katzenschlager R, Perez Lloret S, et al. Update on treatments for nonmotor symptoms of Parkinson's disease-an evidence-based medicine review. Mov Disord. (2019) 34:180-98. doi: $10.1002 / \mathrm{mds} .27602$ 
11. Jankovic J, Goodman I, Safirstein B, Marmon TK, Schenk DB, Koller M, et al. Safety and tolerability of multiple ascending doses of PRX002/RG7935, an anti-alpha-synuclein monoclonal antibody, in patients with Parkinson disease: a randomized clinical trial. JAMA Neurol. (2018) 75:1206-14. doi: 10.1001/jamaneurol.20 18.1487

12. Masliah E, Rockenstein E, Mante M, Crews L, Spencer B, Adame A, et al. Passive immunization reduces behavioral and neuropathological deficits in an alpha-synuclein transgenic model of Lewy body disease. PLoS ONE. (2011) 6:e19338. doi: 10.1371/journal.pone.0019338

13. Poewe W, Seppi K, Tanner CM, Halliday GM, Brundin P, Volkmann J, et al. Parkinson disease. Nat Rev Dis Prim. (2017) 3:17013. doi: 10.1038/nrdp. 2017.13

14. Beach TG, Adler CH, Lue L, Sue LI, Bachalakuri J, Henry-Watson J, et al. Unified staging system for Lewy body disorders: correlation with nigrostriatal degeneration, cognitive impairment and motor dysfunction. Acta Neuropathol. (2009) 117:613-34. doi: 10.1007/s00401-0090538-8

15. Del Tredici K, Braak H. Review: Sporadic Parkinson's disease: development and distribution of alpha-synuclein pathology. Neuropathol Appl Neurobiol. (2016) 42:33-50. doi: 10.1111/nan.12298

16. Shahmoradian SH, Lewis AJ, Genoud C, Hench J, Moors TE, Navarro $\mathrm{PP}$, et al. Lewy pathology in Parkinson's disease consists of crowded organelles and lipid membranes. Nat Neurosci. (2019) 22:1099-109. doi: 10.1038/s41593-019-0423-2

17. Moors TE, Maat CA, Niedieker D, Mona D, Petersen D, TimmermansHuisman E, et al. The subcellular arrangement of alpha-synuclein proteoforms in the Parkinson's disease brain as revealed by multicolor STED microscopy. Acta Neuropathol. (2021) 142:423-48. doi: 10.1007/s00401-021-02329-9

18. Spillantini MG, Schmidt ML, Lee VM, Trojanowski JQ, Jakes R, Goedert M. Alpha-synuclein in Lewy bodies. Nature. (1997) 388:839-40. doi: $10.1038 / 42166$

19. Mahul-Mellier AL, Burtscher J, Maharjan N, Weerens L, Croisier M, Kuttler F, et al. The process of Lewy body formation, rather than simply alphasynuclein fibrillization, is one of the major drivers of neurodegeneration. Proc Natl Acad Sci USA. (2020) 117:4971-82. doi: 10.1073/pnas.19139 04117

20. Rey NL, Steiner JA, Maroof N, Luk KC, Madaj Z, Trojanowski JQ, et al. Widespread transneuronal propagation of $\alpha$-synucleinopathy triggered in olfactory bulb mimics prodromal Parkinson's disease. J Exp Med. (2016) 13:1759-78. doi: 10.1084/jem.20160368

21. Luk KC, Kehm V, Carroll J, Zhang B, O’Brien P, Trojanowski JQ, et al. Pathological alpha-synuclein transmission initiates Parkinson-like neurodegeneration in nontransgenic mice. Science. (2012) 338:949-53. doi: $10.1126 /$ science. 1227157

22. Games D, Seubert P, Rockenstein E, Patrick C, Trejo M, Ubhi K, et al. Axonopathy in an alpha-synuclein transgenic model of Lewy body disease is associated with extensive accumulation of C-terminal-truncated alphasynuclein. Am J Pathol. (2013) 182:940-53. doi: 10.1016/j.ajpath.2012. 11.018

23. Hansen C, Angot E, Bergstrom AL, Steiner JA, Pieri L, Paul G, et al. $\alpha$ synuclein propagates from mouse brain to grafted dopaminergic neurons and seeds aggregation in cultured human cells. J Clin Investig. (2011) 121:715-25. doi: $10.1172 /$ JCI43366

24. Desplats P, Lee HJ, Bae EJ, Patrick C, Rockenstein E, Crews L, et al. Inclusion formation and neuronal cell death through neuron-to-neuron transmission of alpha-synuclein. Proc Natl Acad Sci USA. (2009) 106:13010-5. doi: 10.1073/pnas.0903691106

25. Luk KC, Kehm VM, Zhang B, O’Brien P, Trojanowski JQ, Lee VM. Intracerebral inoculation of pathological alpha-synuclein initiates a rapidly progressive neurodegenerative alpha-synucleinopathy in mice. J Exp Med. (2012) 209:975-86. doi: 10.1084/jem.201 12457

26. Volpicelli-Daley LA, Luk KC, Patel TP, Tanik SA, Riddle DM, Stieber A, et al. Exogenous alpha-synuclein fibrils induce Lewy body pathology leading to synaptic dysfunction and neuron death. Neuron. (2011) 72:57-71. doi: 10.1016/j.neuron.2011.08.033
27. Kim S, Kwon SH, Kam TI, Panicker N, Karuppagounder SS, Lee S, et al. Transneuronal propagation of pathologic alpha-synuclein from the gut to the brain models Parkinson's disease. Neuron. (2019) 103:627-41 e7. doi: 10.1016/j.neuron.2019.05.035

28. Rey NL, Bousset L, George S, Madaj Z, Meyerdirk L, Schulz E, et al. alpha-Synuclein conformational strains spread, seed and target neuronal cells differentially after injection into the olfactory bulb. Acta Neuropathol Commun. (2019) 7:221. doi: 10.1186/s40478-0190859-3

29. Polymeropoulos MH, Lavedan C, Leroy E, Ide SE, Dehejia A, Dutra A, et al. Mutation in the alpha-synuclein gene identified in families with Parkinson's disease. Science. (1997) 276:2045-7. doi: 10.1126/science.276.53 21.2045

30. Singleton AB, Farrer M, Johnson J, Singleton A, Hague S, Kachergus J, et al. Alpha-synuclein locus triplication causes Parkinson's disease. Science. (2003) 302:841. doi: 10.1126/science. 1090278

31. Ibanez P, Bonnet AM, Debarges B, Lohmann E, Tison F, Pollak P, et al. Causal relation between alpha-synuclein gene duplication and familial Parkinson's disease. Lancet. (2004) 364:1169-71. doi: 10.1016/S0140-6736(04) 17104-3

32. Kay DM, Factor SA, Samii A, Higgins DS, Griffith A, Roberts JW, et al. Genetic association between alpha-synuclein and idiopathic Parkinson's disease. Am J Med Genet Part B Neuropsychiatric Genet. (2008) 147B:1222-30. doi: 10.1002/ajmg.b.30758

33. Bekris LM, Mata IF, Zabetian CP. The genetics of Parkinson disease. J Geriatr Psychiatry Neurol. (2010) 23:228-42. doi: 10.1177/0891988710383572

34. Devine MJ, Gwinn K, Singleton A, Hardy J. Parkinson's disease and alphasynuclein expression. Mov Dis. (2011) 26:2160-8. doi: 10.1002/mds.23948

35. Li JY, Englund E, Holton JL, Soulet D, Hagell P, Lees AJ, et al. Lewy bodies in grafted neurons in subjects with Parkinson's disease suggest host-to-graft disease propagation. Nat Med. (2008) 14:501-3. doi: 10.1038/ nm1746

36. Kordower JH, Chu Y, Hauser RA, Freeman TB, Olanow CW. Lewy body-like pathology in long-term embryonic nigral transplants in Parkinson's disease. Nat Med. (2008) 14:504-6. doi: 10.1038/nm1747

37. Wilson H, Dervenoulas G, Pagano G, Koros C, Yousaf T, Picillo M, et al. Serotonergic pathology and disease burden in the premotor and motor phase of A53T alpha-synuclein parkinsonism: a cross-sectional study. Lancet Neurol. (2019) 18:748-59. doi: 10.1016/S1474-4422(19) 30140-1

38. Knudsen K, Fedorova TD, Hansen AK, Sommerauer M, Otto M, Svendsen $\mathrm{KB}$, et al. In-vivo staging of pathology in REM sleep behaviour disorder: a multimodality imaging case-control study. Lancet Neurol. (2018) 17:618-28. doi: 10.1016/S1474-4422(18)30162-5

39. Dehay B, Bourdenx M, Gorry P, Przedborski S, Vila M, Hunot S, et al. Targeting alpha-synuclein for treatment of Parkinson's disease: mechanistic and therapeutic considerations. Lancet Neurol. (2015) 14:855-66. doi: 10.1016/S1474-4422(15)00006-X

40. Masliah E, Rockenstein E, Adame A, Alford M, Crews L, Hashimoto $\mathrm{M}$, et al. Effects of alpha-synuclein immunization in a mouse model of Parkinson's disease. Neuron. (2005) 46:857-68. doi: 10.1016/j.neuron.2005. 05.010

41. Games D, Valera E, Spencer B, Rockenstein E, Mante M, Adame $A$, et al. Reducing C-terminal-truncated alpha-synuclein by immunotherapy attenuates neurodegeneration and propagation in Parkinson's disease-like models. J Neurosci. (2014) 34:9441-54. doi: 10.1523/JNEUROSCI.5314-13.2014

42. Spencer B, Valera E, Rockenstein E, Overk C, Mante M, Adame $\mathrm{A}$, et al. Anti-alpha-synuclein immunotherapy reduces alpha-synuclein propagation in the axon and degeneration in a combined viral vector and transgenic model of synucleinopathy. Acta Neuropathol Commun. (2017) 5:7. doi: 10.1186/s40478-016-0410-8

43. Tran HT, Chung CH, Iba M, Zhang B, Trojanowski JQ, Luk KC, et al. Alpha-synuclein immunotherapy blocks uptake and templated propagation of misfolded alpha-synuclein and neurodegeneration. Cell Rep. (2014) 7:205465. doi: 10.1016/j.celrep.2014.05.033

44. Kallab M, Herrera-Vaquero M, Johannesson M, Eriksson F, Sigvardson J, Poewe W, et al. Region-specific effects of immunotherapy with 
antibodies targeting alpha-synuclein in a transgenic model of synucleinopathy. Front Neurosci. (2018) 12:452. doi: 10.3389/fnins.2018. 00452

45. Schenk DB, Koller M, Ness DK, Griffith SG, Grundman M, Zago W, et al. First-in-human assessment of PRX002, an anti-alpha-synuclein monoclonal antibody, in healthy volunteers. Mov Disord. (2017) 32:211-8. doi: $10.1002 / \mathrm{mds} .26878$

46. PPMI. A Landmark Study of Parkinson's Disease. (2018). Available online at: https://www.ppmi-info.org/ (accessed May 2021)

47. Simuni T, Siderowf A, Lasch S, Coffey CS, Caspell-Garcia C, Jennings D, et al. Longitudinal change of clinical and biological measures in early Parkinson's disease: Parkinson's progression markers initiative cohort. Mov Disord. (2018) 33:771-82. doi: $10.1002 / \mathrm{mds} .27361$

48. ClinicalTrials.gov. NCT03100149: A Study to Evaluate the Efficacy of Prasinezumab (RO7046015/PRX002) in Participants with Early Parkinson's Disease (PASADENA). (2017). Available online at: https://clinicaltrials.gov/ ct 2/show/NCT03100149?term=prasinezumab\&draw $=2 \&$ rank $=1 \quad$ (accessed May 2021)

49. Goetz CG, Tilley BC, Shaftman SR, Stebbins GT, Fahn S, MartinezMartin P, et al. Movement Disorder Society-sponsored revision of the Unified Parkinson's Disease Rating Scale (MDS-UPDRS): scale presentation and clinimetric testing results. Mov Disord. (2008) 23:2129-70. doi: $10.1002 / \mathrm{mds} .22340$

50. Taylor KI, Staunton H, Lipsmeier F, Nobbs D, Lindemann M. Outcome measures based on digital health technology sensor data: data- and patient-centric approaches. NPJ Digit Med. (2020) 3:97. doi: 10.1038/s41746-020-0305-8

51. Lipsmeier F, Simillion C, Bamdadian A, Smith A, Schobel S, Gossens C, et al. Reliability, feasibility and validity of a novel digital monitoring platform assessing cognitive and motor symptoms in people with Stage I and II Huntington's disease (HD). Neurotherapeutics. (2019) 16:1350-90. doi: 10.1007/s13311-019-00788-3

52. Marek K, Chowdhury S, Siderowf A, Coffey CS, Caspell-Garcia CJ, Simuni T, et al. The Parkinson's progression markers initiative (PPMI) - establishing a PD biomarker cohort. Ann Clin Transl Neurol. (2018) 5:1460-77. doi: $10.1002 / \mathrm{acn} 3.644$

53. Horvath K, Aschermann Z, Kovacs M, Makkos A, Harmat M, Janszky J, et al. Minimal clinically important differences for the experiences of daily living parts of movement disorder society-sponsored unified Parkinson's disease rating scale. Mov Disord. (2017) 32:789-93. doi: 10.1002/mds. 26960

54. Parkinson Progression Marker Initiative. The Parkinson progression marker initiative (PPMI). Prog Neurobiol. (2011) 95:629-35. doi: 10.1016/j.pneurobio.2011.09.005

55. Stuart EA, Lee BK, Leacy FP. Prognostic score-based balance measures can be a useful diagnostic for propensity score methods in comparative effectiveness research. J Clin Epidemiol. (2013) 66(Suppl. 8):S84-90.e1. doi: 10.1016/j.jclinepi.2013.01.013

56. Rascol O, Fitzer-Attas CJ, Hauser R, Jankovic J, Lang A, Langston JW, et al. A double-blind, delayed-start trial of rasagiline in Parkinson's disease (the ADAGIO study): prespecified and post-hoc analyses of the need for additional therapies, changes in UPDRS scores, and non-motor outcomes. Lancet Neurol. (2011) 10:415-23. doi: 10.1016/S1474-4422(11) 70073-4

57. Parkinson Study Group. A controlled trial of rasagiline in early Parkinson disease: the TEMPO Study. Arch Neurol. (2002) 59:1937-43. doi: 10.1001/archneur.59.12.1937

58. Kalia LV, Kalia SK, Lang AE. Disease-modifying strategies for Parkinson's disease. Mov Disord. (2015) 30:1442-50. doi: 10.1002/mds.26354

59. Mollenhauer B, Trautmann E, Sixel-Doring F, Wicke T, Ebentheuer J, Schaumburg $\mathrm{M}$, et al. Nonmotor and diagnostic findings in subjects with de novo Parkinson disease of the DeNoPa cohort. Neurology. (2013) 81:1226-34. doi: 10.1212/WNL.0b013e3182a6cbd5

60. A controlled trial of lazabemide (Ro 19-6327) in levodopa-treated Parkinson's disease. Parkinson Study Group. Arch Neurol. (1994) 51:342-7. doi: 10.1001/archneur.1994.00540160036006
61. Teo KC, Ho SL. Monoamine oxidase-B (MAO-B) inhibitors: implications for disease-modification in Parkinson's disease. Transl Neurodegener. (2013) 2:19. doi: 10.1186/2047-9158-2-19

62. Qamar MA, Sauerbier A, Politis M, Carr H, Loehrer P, Chaudhuri KR. Presynaptic dopaminergic terminal imaging and non-motor symptoms assessment of Parkinson's disease: evidence for dopaminergic basis? NPJ Parkinsons Dis. (2017) 3:5. doi: 10.1038/s41531-016-0006-9

63. Connolly BS, Lang AE. Pharmacological treatment of Parkinson disease: a review. JAMA. (2014) 311:1670-83. doi: 10.1001/jama.2014.3654

64. Holden SK, Finseth T, Sillau SH, Berman BD. Progression of MDS-UPDRS scores over five years in de novo Parkinson disease from the Parkinson's progression markers initiative cohort. Mov Disord Clin Pract. (2018) 5:47-53. doi: $10.1002 / \mathrm{mdc} 3.12553$

65. Pagano G, Niccolini F, Politis M. Imaging in Parkinson's disease. Clin Med. (2016) 16:371-5. doi: 10.7861/clinmedicine.16-4-371

66. Nandhagopal R, Kuramoto L, Schulzer M, Mak E, Cragg J, McKenzie J, et al. Longitudinal evolution of compensatory changes in striatal dopamine processing in Parkinson's disease. Brain. (2011) 134(Pt 11):3290-8. doi: 10.1093/brain/awr233

67. Schwarz J, Storch A, Koch W, Pogarell O, Radau PE, Tatsch K. Loss of dopamine transporter binding in Parkinson's disease follows a single exponential rather than linear decline. J Nucl Med. (2004) 45:1694-7.

68. Maetzler W, Domingos J, Srulijes K, Ferreira JJ, Bloem BR. Quantitative wearable sensors for objective assessment of Parkinson's disease. Mov Disord. (2013) 28:1628-37. doi: $10.1002 / \mathrm{mds} .25628$

69. Ossig C, Antonini A, Buhmann C, Classen J, Csoti I, Falkenburger $\mathrm{B}$, et al. Wearable sensor-based objective assessment of motor symptoms in Parkinson's disease. J Neural Transm. (2016) 123:57-64. doi: 10.1007/s00702-015-1439-8

70. Lipsmeier F, Fernandez Garcia I, Wolf D, Kilchenmann T, Scotland A, SchjodtEriksen J, et al. Successful Passive Monitoring of Early-Stage Parkinson's Disease Patient Mobility in Phase I RG7935/PRX002 Clinical Trial with Smartphone Sensors. Basel: MDS (2017).

71. Lipsmeier F, Taylor KI, Kilchenmann T, Wolf D, Scotland A, Schjodt-Eriksen J, et al. Evaluation of smartphone-based testing to generate exploratory outcome measures in a phase 1 Parkinson's disease clinical trial. Mov Disord. (2018) 33:1287-97. doi: 10.1002/mds.27376

72. Lipsmeier F, Taylor KI, Postuma R, Kilchenmann T, Wolf D, Zhang $\mathrm{Y}$, et al. Preliminary validation of a novel, comprehensive digital biomarker smartphone application to assess motor symptoms in recently diagnosed Parkinson patients (P4.7-005). Neurology. (2019) 92.

73. Jankovic J. Parkinson's disease: clinical features and diagnosis. $J$ Neurol Neurosurg Psychiatry. (2008) 79:368-76. doi: 10.1136/jnnp.2007. 131045

74. Wang N, Gibbons CH, Lafo J, Freeman R. Alpha-synuclein in cutaneous autonomic nerves. Neurology. (2013) 81:1604-10. doi: 10.1212/WNL.0b013e3182a9f449

75. Donadio V, Incensi A, Leta V, Giannoccaro MP, Scaglione C, Martinelli P, et al. Skin nerve alpha-synuclein deposits: a biomarker for idiopathic Parkinson disease. Neurology. (2014) 82:1362-9. doi: 10.1212/WNL.00000000000 00316

76. Horvath K, Aschermann Z, Acs P, Deli G, Janszky J, Komoly S, et al. Minimal clinically important difference on the Motor Examination part of MDS-UPDRS. Parkinsonism Relat Disord. (2015) 21:1421-6. doi: 10.1016/j.parkreldis.2015.10.006

77. Mischley LK, Lau RC, Weiss NS. Use of a self-rating scale of the nature and severity of symptoms in Parkinson's Disease (PRO-PD): Correlation with quality of life and existing scales of disease severity. NPJ Parkinsons Dis. (2017) 3:20. doi: 10.1038/s41531-017-0021-5

78. Poewe W, Espay AJ. Long duration response in Parkinson's disease: levodopa revisited. Brain J Neurol. (2020) 143:2332-5. doi: 10.1093/brain/aw aa226

79. Hustad E, Aasly JO. Clinical and imaging markers of prodromal Parkinson's disease. Front Neurol. (2020) 11:395. doi: 10.3389/fneur.2020. 00395 
80. Campbell CM, Gilron I, Doshi T, Raja S. Designing and conducting proofof-concept chronic pain analgesic clinical trials. Pain Rep. (2019) 4:e697. doi: 10.1097/PR9.0000000000000697

Conflict of Interest: GP, FB, KT, BR, JA-C, AV, MM, TN, HS, MB, SO, RD, DU, and ABon are employees of F. Hoffmann-La Roche Ltd. ABou is an employee of Idorsia Pharmaceuticals Ltd. AP is an employee of Feetme SAS. JS is an employee of Prevail Therapeutics. GK, WZ, DN, and MK are employees of Prothena Biosciences Inc. The authors declare that F. Hoffmann-La Roche Ltd was the sponsor of the study. F. Hoffmann-La Roche Ltd and Prothena Corporation plc were the funders of the study. F. Hoffmann-La Roche Ltd and Prothena Corporation plc were involved in the study design, collection, analysis, interpretation of data, the writing of this article and the decision to submit it for publication.

The remaining authors declare that the research was conducted in the absence of any commercial or financial relationships that could be construed as a potential conflict of interest.
Publisher's Note: All claims expressed in this article are solely those of the authors and do not necessarily represent those of their affiliated organizations, or those of the publisher, the editors and the reviewers. Any product that may be evaluated in this article, or claim that may be made by its manufacturer, is not guaranteed or endorsed by the publisher.

Copyright (C) 2021 Pagano, Boess, Taylor, Ricci, Mollenhauer, Poewe, Boulay, Anzures-Cabrera, Vogt, Marchesi, Post, Nikolcheva, Kinney, Zago, Ness, Svoboda, Britschgi, Ostrowitzki, Simuni, Marek, Koller, Sevigny, Doody, Fontoura, Umbricht, Bonni and PASADENA Investigators and Prasinezumab Study Group. This is an open-access article distributed under the terms of the Creative Commons Attribution License (CC BY). The use, distribution or reproduction in other forums is permitted, provided the original author(s) and the copyright owner(s) are credited and that the original publication in this journal is cited, in accordance with accepted academic practice. No use, distribution or reproduction is permitted which does not comply with these terms. 